\title{
ERROR BOUNDS FOR FINITE-DIFFERENCE METHODS FOR RUDIN-OSHER-FATEMI IMAGE SMOOTHING*
}

\author{
JINGYUE WANG ${ }^{\dagger}$ AND BRADLEY J. LUCIER ${ }^{\ddagger}$
}

\begin{abstract}
We bound the difference between the solution to the continuous Rudin-Osher-Fatemi image smoothing model and the solutions to various finite-difference approximations to this model. These bounds apply to "typical" images, i.e., images with edges or with fractal structure. These are the first bounds on the error in numerical methods for ROF smoothing.
\end{abstract}

Key words. Total variation, bounded variation, variational problems, finite-difference methods, image processing.

AMS subject classifications. 65N06, 65N12, 94A08

1. Introduction. Image noise removal based on total variation smoothing was introduced by Rudin, Osher, and Fatemi in [13]. Under this ROF model, one supposes a "true" image $f$ defined on $\Omega=[0,1]^{2}$ and a "corrupted" image $g$ derived from $f$ (by adding noise, etc.) with $\|f-g\|_{L^{2}(\Omega)}^{2}=\sigma^{2}$. In an attempt to reconstruct $f$ from $g$, one calculates a "smoothed" image $u$ that minimizes

$$
|v|_{\mathrm{BV}(\Omega)}=\int_{\Omega}|D v| \text { subject to the constraint }\|v-g\|_{L^{2}(\Omega)}^{2} \leq \sigma^{2} .
$$

(Precise definitions are given later.) We deal with the equivalent problem: If we calculate $\bar{g}$, the average of $g$ on $\Omega$, then for any $\sigma$ with

$$
\sigma^{2}<\|g-\bar{g}\|_{L^{2}(\Omega)}^{2}
$$

there exists a unique $\lambda>0$ such that the minimizer of (1.1) is the minimizer $u$ of the functional

$$
E(v)=\frac{1}{2 \lambda}\|v-g\|_{L^{2}(\Omega)}^{2}+|v|_{\mathrm{BV}(\Omega)} .
$$

Here $\lambda$ is a positive parameter that balances the relative importance of the smoothness of the minimizer (important when $\lambda$ is large) and the $L^{2}(\Omega)$ distance between the minimizer and the initial data (important when $\lambda$ is small). About the same time, Bouman and Sauer [1] proposed a discrete version of (1.2) in the context of tomography.

Practically one discretizes $E(\cdot)$ to compute the minimizer of the discrete functional $E_{h}(\cdot)$. We assume the discrete corrupted image $g^{h}$ of resolution $N \times N(N=1 / h)$ is simply the piecewise constant projection of the continuous corrupted image $g$, and define the discrete functional

$$
E_{h}\left(v^{h}\right)=\frac{1}{2 \lambda} \sum_{i}\left|v_{i}^{h}-g_{i}^{h}\right|^{2} h^{2}+J_{h}\left(v^{h}\right)
$$

\footnotetext{
*This work was partially supported by the Office of Naval Research, Contract N00014-91-J-1152, and the Institute for Mathematics and its Applications, the University of Minnesota.

${ }^{\dagger}$ Department of Mathematics, University of Georgia, Athens, Georgia 30602 (jwang@math.uga.edu).

‡Department of Mathematics, Purdue University, West Lafayette, Indiana 47907-2067 (lucier@math.purdue.edu).
} 
where $J_{h}$ is a discretized total variation. The most-commonly used $J_{h}$ is the discrete variation $J_{++}$used in [13]

$$
J_{++}\left(v^{h}\right)=\sum_{i} \sqrt{\left(\frac{\left(v_{i+(1,0)}^{h}-v_{i}^{h}\right)}{h}\right)^{2}+\left(\frac{v_{i+(0,1)}^{h}-v_{i}^{h}}{h}\right)^{2}} h^{2} .
$$

Efficient algorithms have been developed to compute the discrete minimizer([2], [6], [3], [5]).

In this paper, we study the relationship between the minimizer $u$ of $E(\cdot)$ and the discrete minimizer $u^{h}$ of $E_{h}(\cdot)$. It is well known that $E_{h} \Gamma$-converges to $E$ in $L^{1}$. As a direct deduction $u^{h}$ tends to $u$ in $L^{1}$. Assuming the discrete variation $J_{h}$ satisfies certain conditions that we explain later, we give a bound of the $L^{2}$ norm of the difference between $u$ and $u^{h}$ in Theorem 4.2 in Section 4 .

Because the ROF model is often applied to images, an analysis of the error between solutions of discrete approximations and the solution of the continuous model itself should apply to functions modelling images. "Typical" natural images have little smoothness, because of intensity discontinuities at the edges of objects and the fractal structure of many objects themselves (the leaves in a tree, hair, etc.). Our results apply to functions in the Lipschitz spaces $\operatorname{Lip}\left(\alpha, L^{2}(\Omega)\right)$, which contain functions with, roughly speaking, $\alpha$ "derivatives" in $L^{2}(\Omega)$. Here $0<\alpha \leq 1 / 2$ for "images with edges": $f \in \operatorname{BV}(\Omega) \cap L^{\infty}(\Omega)$ implies $f \in \operatorname{Lip}\left(1 / 2, L^{2}(\Omega)\right)$, while functions with fractal structure usually have $\alpha<1 / 2$, see [7].

Our convergence results in Section 4 are proved for (1.3) with $J_{h}=J_{*}$, a discrete variation obtained by symmetrizing $J_{++}$. Nonetheless, our approach is quite general, and in Section 5 we obtain the same results for $J_{U}$, an "upwind" discrete variation formulated in [12]. We remark that an iterative method for minimizing (1.3) with $J_{h}=J_{U}$ was given in [5].

While the ROF model has proved to be tremendously influential, and has been the base of further algorithms in image processing, we know of no other results that bound the difference between the solutions of the continuous problems and its finitedifference approximations. A finite element method applied to the time-dependent gradient descent problem associated with (1.2) was studied in [10]; we note that their Theorem 4 requires the initial data $u_{0}$ to have two continuous derivatives on $\bar{\Omega}$ so it does not apply to "typical" natural images with edges.

The rest of this section introduces the notation for, and basic properties of, operators and functionals on continuous and discrete functions. In Section 2 we compare discrete and continuous variational functionals. In Section 3 we note some properties of the minimizers of $E(\cdot)$ and $E_{h}(\cdot)$ that we use in Section 4 to first bound the discrete and continuous functionals at their respective minimizers and then to bound the $L^{2}$ difference between the discrete and continuous minimizers themselves. In Section 5, we prove a number of lemmas for the "upwind" discretization of the BV semi-norm that allows us to prove similar error bounds for the discrete minimizer of the "upwind" scheme. Finally, Section 6 summarizes our results and points to variations that appear elsewhere.

1.1. Basic notations. We consider the usual $L^{p}(\Omega)$ spaces on $\Omega:=[0,1]^{2} \subset \mathbb{R}^{2}$, with

$$
\|v\|_{L^{p}(\Omega)}:=\left(\int_{\Omega}|v|^{p}\right)^{\frac{1}{p}}
$$


for $1 \leq p<\infty$ and

$$
\|v\|_{L^{\infty}(\Omega)}=\operatorname{ess}_{x \in \Omega} \sup |v(x)| .
$$

We consider the discrete set $\Omega^{h}$ to be the set of all pairs $i=\left(i_{1}, i_{2}\right) \in \mathbb{Z}^{2}, \mathbb{Z}$ the integers, with $0 \leq i_{1}, i_{2}<N, h=1 / N$, and we refer to functions defined on $\Omega^{h}$ as discrete functions. So for discrete functions $v^{h}=v_{i}^{h}$, we define the discrete $L^{p}\left(\Omega^{h}\right)$ norms

$$
\left\|v^{h}\right\|_{L^{p}\left(\Omega^{h}\right)}:=\left(\sum_{i \in \Omega^{h}}\left|v_{i}^{h}\right|^{p} h^{2}\right)^{\frac{1}{p}} \text { for } 1 \leq p<\infty
$$

and

$$
\left\|v^{h}\right\|_{L^{\infty}\left(\Omega^{h}\right)}=\max _{i \in \Omega^{h}}\left|v_{i}^{h}\right| .
$$

In later definitions, we assume the reader will apply the usual modifications when $p=\infty$.

We define the translation operator for discrete functions by

$$
\left(T_{\ell}\left(v^{h}\right)\right)_{i}:=v_{i+\ell}^{h} \quad \text { for any } \ell=\left(\ell_{1}, \ell_{2}\right) \in \mathbb{Z}^{2} .
$$

To measure the size of a translation, we introduce

$$
|\ell|=\max \left(\left|\ell_{1}\right|,\left|\ell_{2}\right|\right)
$$

Similarly,

$$
\left(\mathcal{T}_{\tau} v\right)(x)=v(x+\tau) \quad \text { for any } \tau=\left(\tau_{1}, \tau_{2}\right) \in \mathbb{R}^{2}
$$

and, for translations, we set

$$
|\tau|=\max \left(\left|\tau_{1}\right|,\left|\tau_{2}\right|\right) .
$$

We often need to extend $v \in L^{p}(\Omega)$ and $v^{h} \in L^{p}\left(\Omega^{h}\right)$ to all of $\mathbb{R}^{2}$ and $\mathbb{Z}^{2}$, respectively; we denote the extensions by Ext $v$ and $\operatorname{Ext}_{h} v^{h}$. For $v \in L^{p}(\Omega)$, we use the following procedure. First,

$$
\operatorname{Ext} v(x)=v(x), \quad x \in \Omega .
$$

We then reflect horizontally across the line $x_{1}=1$,

$$
\operatorname{Ext} v\left(x_{1}, x_{2}\right)=\operatorname{Ext} v\left(2-x_{1}, x_{2}\right), \quad 1 \leq x_{1} \leq 2,0 \leq x_{2} \leq 1,
$$

and reflect again vertically across the line $x_{2}=1$,

$$
\operatorname{Ext} v\left(x_{1}, x_{2}\right)=\operatorname{Ext} v\left(x_{1}, 2-x_{2}\right), \quad 0 \leq x_{1} \leq 2,1 \leq x_{2} \leq 2 .
$$

Having defined Ext $v$ on $2 \Omega$, we then extend Ext $v$ periodically on all of $\mathbb{R}^{2}$.

We use a similar constructions for discrete functions $v^{h}$. First we extend $v^{h}$ to

$$
2 \Omega^{h}:=\left\{i=\left(i_{1}, i_{2}\right) \in \mathbb{Z}^{2} \mid 0 \leq i_{1}, i_{2}<2 N\right\}
$$


as follows:

$$
\operatorname{Ext}_{h} v_{i}^{h}=v_{i}^{h}, \quad i \in \Omega^{h}
$$

then we reflect horizontally

$$
\operatorname{Ext}_{h} v_{\left(i_{1}, i_{2}\right)}^{h}=\operatorname{Ext}_{h} v_{\left(2 N-i_{1}-1, i_{2}\right)}^{h}, \quad N \leq i_{1}<2 N, 0 \leq i_{2}<N,
$$

and then vertically

$$
\operatorname{Ext}_{h} v_{\left(i_{1}, i_{2}\right)}^{h}=\operatorname{Ext}_{h} v_{\left(i_{1}, 2 N-i_{1}-1\right)}^{h}, \quad 0 \leq i_{1}<2 N, N \leq i_{2}<2 N .
$$

Now that $\operatorname{Ext}_{h} v^{h}$ is defined on $2 \Omega^{h}$, we extend it periodically to all of $\mathbb{Z}^{2}$. Note that with this definition, the value of $\operatorname{Ext}_{h} v^{h}$ at any point immediately "outside" $\Omega^{h}$ is the same as the value of $v^{h}$ at the closest point "inside" $\Omega^{h}$.

For $v \in L^{p}(\Omega)$ we define the (first-order) $L^{p}(\Omega)$ modulus of smoothness by

$$
\omega(v, t)_{L^{p}(\Omega)}=\sup _{\tau \in \mathbb{R}^{2},|\tau|<t}\left(\int_{x, x+\tau \in \Omega}|v(x+\tau)-v(x)|^{p} d x\right)^{\frac{1}{p}} .
$$

We also define

$$
\omega(\operatorname{Ext} v, t)_{L^{p}(2 \Omega)}:=\sup _{\tau \in \mathbb{R}^{2},|\tau|<t}\left\|\mathcal{T}_{\tau} \operatorname{Ext} v-\operatorname{Ext} v\right\|_{L^{p}(2 \Omega)} .
$$

The Lipschitz spaces $\operatorname{Lip}\left(\alpha, L^{p}(\Omega)\right)$ consist of all functions $v$ for which

$$
|v|_{\operatorname{Lip}\left(\alpha, L^{p}(\Omega)\right)}:=\sup _{t>0} t^{-\alpha} \omega(v, t)_{L^{p}(\Omega)}<\infty ;
$$

we set

$$
\|v\|_{\operatorname{Lip}\left(\alpha, L^{p}(\Omega)\right)}:=\|v\|_{L^{p}(\Omega)}+|v|_{\operatorname{Lip}\left(\alpha, L^{p}(\Omega)\right)} .
$$

We also need a discrete modulus of smoothness. The discrete $L^{p}\left(\Omega^{h}\right)$ modulus of smoothness is

$$
\omega\left(v^{h}, m\right)_{L^{p}\left(\Omega^{h}\right)}:=\sup _{\ell \in \mathbb{Z}^{2},|\ell| \leq m}\left(\sum_{i, i+\ell \in \Omega^{h}}\left|v_{i+\ell}^{h}-v_{i}^{h}\right|^{p} h^{2}\right)^{\frac{1}{p}} .
$$

For $\operatorname{Ext}_{h} v^{h}$ we define similarly

$$
\omega\left(\operatorname{Ext}_{h} v^{h}, m\right)_{L^{p}\left(2 \Omega^{h}\right)}=\sup _{\ell \in \mathbb{Z}^{2},|\ell| \leq m}\left\|T_{\ell} \operatorname{Ext}_{h} v^{h}-\operatorname{Ext}_{h} v^{h}\right\|_{L^{p}\left(2 \Omega^{h}\right)} .
$$

We have the following relationship between moduli of smoothness and our extension operators.

Lemma 1.1 (Whitney extension). For all $1 \leq p \leq \infty$ there exists a constant $C$ such that for all $v \in L^{p}(\Omega)$ and $v^{h} \in L^{p}\left(\Omega^{h}\right)$

$$
\left\|\mathcal{T}_{\tau} \operatorname{Ext} v-\operatorname{Ext} v\right\|_{L^{p}(2 \Omega)} \leq C \omega(v,|\tau|)_{L^{p}(\Omega)} \quad \tau \in \mathbb{R}^{2}
$$

and

$$
\left\|T_{\ell} \operatorname{Ext}_{h} v^{h}-\operatorname{Ext}_{h} v^{h}\right\|_{L^{p}\left(2 \Omega^{h}\right)} \leq C \omega\left(v^{h},|\ell|\right)_{L^{p}\left(\Omega^{h}\right)} \quad \ell \in \mathbb{Z}^{2}
$$


Moreover, for all positive $t \in \mathbb{R}, m \in \mathbb{Z}$ we have

$$
\omega(\operatorname{Ext} v, t)_{L^{p}(2 \Omega)} \leq C \omega(v, t)_{L^{p}(\Omega)}
$$

and

$$
\omega\left(\operatorname{Ext}_{h} v^{h}, m\right)_{L^{p}\left(2 \Omega^{h}\right)} \leq C \omega\left(v^{h}, m\right)_{L^{p}\left(\Omega^{h}\right)} .
$$

Proof. Inequalities (1.4) and (1.6) follow because our extension satisfies the Whitney extension theorem [8], page 182. Inequalities (1.5) and (1.7) are a form of a discrete Whitney extension theorem that can be proved along the lines of the proof of the Whitney extension theorem given in [8].

1.2. Variation functionals. The variation of a function $v \in L^{1}(\Omega)$ is defined as follows. We consider functions $\phi$ in the space of $C^{1}$ functions from $\Omega$ to $\mathbb{R}^{2}$ with compact support, i.e., $\left[C_{0}^{1}(\Omega)\right]^{2}$. The variation of a function $v \in L^{1}(\Omega)$ is then defined to be

$$
|v|_{\mathrm{BV}(\Omega)}:=\int_{\Omega}|D v|:=\sup _{\phi \in\left[C_{0}^{1}(\Omega)\right]^{2},|\phi| \leq 1 \text { pointwise }} \int_{\Omega} v \nabla \cdot \phi .
$$

We note that if $v$ is in the Sobolev space $W^{1,1}(\Omega)$, so that its first distributional derivatives are in $L^{1}(\Omega)$, then

$$
|v|_{\mathrm{BV}(\Omega)}=\int_{\Omega}|\nabla v| .
$$

We need discrete analogues of the variation of a function. For $\oplus$ and $\ominus$ independently taking values in the set $\{+,-\}$ and any discrete function $v^{h}$ we define

$$
J_{\oplus \ominus}\left(v^{h}\right):=\sum_{i \in \Omega^{h}} \sqrt{\left(\frac{\operatorname{Ext}_{h} v_{i \oplus(1,0)}^{h}-\operatorname{Ext}_{h} v_{i}^{h}}{h}\right)^{2}+\left(\frac{\operatorname{Ext}_{h} v_{i \ominus(0,1)}^{h}-\operatorname{Ext}_{h} v_{i}^{h}}{h}\right)^{2}} h^{2} .
$$

We note that the sum is over $i \in \Omega^{h}$, and $\operatorname{Ext}_{h} v_{i}^{h}=v_{i}^{h}$ for all $i \in \Omega^{h}$.

Having defined $J_{++}\left(v^{h}\right), J_{+-}\left(v^{h}\right), J_{-+}\left(v^{h}\right)$, and $J_{--}\left(v^{h}\right)$, we define for any nonnegative $a, b, c$, and $d$ with $a+b+c+d=1$

$$
J_{h}\left(v^{h}\right)=a J_{++}\left(v^{h}\right)+b J_{+-}\left(v^{h}\right)+c J_{-+}\left(v^{h}\right)+d J_{--}\left(v^{h}\right)
$$

and define the special "isotropic" discrete variation

$$
J_{*}\left(v^{h}\right):=\frac{1}{4}\left(J_{++}\left(v^{h}\right)+J_{+-}\left(v^{h}\right)+J_{-+}\left(v^{h}\right)+J_{--}\left(v^{h}\right)\right) ;
$$

$J_{*}$ is invariant under rotations of $\Omega^{h}$ by 90 degrees, or under horizontal or vertical reflections.

At times we consider discrete variational functionals for discrete functions defined on $2 \Omega^{h}$; for these purposes we denote by $J_{\oplus \ominus}^{\Omega^{h}}\left(v^{h}\right)$ the discrete variation defined in (1.8) and $J_{\oplus \ominus}^{2 \Omega^{h}}\left(\operatorname{Ext}_{h} v^{h}\right)$ the corresponding sum over $2 \Omega^{h}$; similarly we write

$$
J_{h}^{\Omega^{h}}\left(v^{h}\right)=J_{h}\left(v^{h}\right) \text { and } J_{*}^{\Omega^{h}}\left(v^{h}\right)=J_{*}\left(v^{h}\right)
$$


and we use the notation $J_{h}^{2 \Omega^{h}}\left(\operatorname{Ext}_{h} v^{h}\right)$ for

$$
a J_{++}^{2 \Omega^{h}}\left(\operatorname{Ext}_{h} v^{h}\right)+b J_{+-}^{2 \Omega^{h}}\left(\operatorname{Ext}_{h} v^{h}\right)+c J_{-+}^{2 \Omega^{h}}\left(\operatorname{Ext}_{h} v^{h}\right)+d J_{--}^{2 \Omega^{h}}\left(\operatorname{Ext}_{h} v^{h}\right)
$$

and $J_{*}^{2 \Omega^{h}}\left(\operatorname{Ext}_{h} v^{h}\right)$ for the corresponding sum with $a=b=c=d=1 / 4$.

We have the following relationships between continuous and discrete variations of functions and the continuous and discrete extension operators.

Lemma 1.2 (TV symmetry). For any discrete function $v^{h}$,

$$
J_{\oplus \ominus}^{2 \Omega^{h}}\left(\operatorname{Ext}_{h} v^{h}\right)=4 J_{*}^{\Omega^{h}}\left(v^{h}\right) .
$$

Thus, we have

$$
J_{*}^{2 \Omega^{h}}\left(\operatorname{Ext}_{h} v^{h}\right)=4 J_{*}^{\Omega^{h}}\left(v^{h}\right)
$$

and for any $\ell \in \mathbb{Z}^{2}$

$$
J_{*}^{2 \Omega^{h}}\left(T_{\ell} \operatorname{Ext}_{h} v^{h}\right)=4 J_{*}^{\Omega^{h}}\left(v^{h}\right) .
$$

Similarly, for any $v \in \mathrm{BV}(\Omega)$, we have

$$
|\operatorname{Ext} v|_{\mathrm{BV}(2 \Omega)}=4|v|_{\mathrm{BV}(\Omega)} .
$$

Proof. Relation (1.10) follows because we construct $\operatorname{Ext}_{h} v^{h}$ by reflection across the boundaries of $\Omega^{h}$, and, for example, $J_{++}\left(\operatorname{Ext} v^{h}\right)$ on the lower-left quadrant of $2 \Omega^{h}$ is equal to $J_{--}\left(\operatorname{Ext} v^{h}\right)$ on the upper right quadrant of $2 \Omega^{h}$.

The next two relations follow immediately from (1.10).

Relation (1.13) is a consequence of the fact that we introduce no variation in Ext $v$ along the original boundary of $\Omega$ by reflecting $v$ across that boundary.

We also define a discrete "anisotropic" variation that is analogous to the $W^{1,1}(\Omega)$ Sobolev seminorm:

$$
\left|v^{h}\right|_{W^{1,1}\left(\Omega^{h}\right)}=\sum_{i \in \Omega^{h}}\left\{\left|\frac{\operatorname{Ext}_{h} v_{i+(1,0)}^{h}-\operatorname{Ext}_{h} v_{i}^{h}}{h}\right|+\left|\frac{\operatorname{Ext}_{h} v_{i+(0,1)}^{h}-\operatorname{Ext}_{h} v_{i}^{h}}{h}\right|\right\} h^{2} .
$$

Because, for nonnegative $x_{i}$

$$
\sum_{i=1}^{n} x_{i}^{2} \leq\left(\sum_{i=1}^{n} x_{i}\right)^{2} \leq n \sum_{i=1}^{n} x_{i}^{2}
$$

$\left|v^{h}\right|_{W^{1,1}\left(\Omega^{h}\right)}$ is equivalent to any $J_{h}\left(v^{h}\right)$ (take $\left.n=2\right)$ and, a fortiori, to any particular $J_{\oplus \ominus}\left(v^{h}\right)$. Thus we have the following lemma.

Lemma 1.3 (Discrete TV equivalence). $J_{h}$ is equivalent to $|\cdot|_{W^{1,1}\left(\Omega^{h}\right)}$. To be precise, there exist positive constants $C_{1}$ and $C_{2}$, such that for any discrete function $v^{h}$

$$
C_{1}\left|v^{h}\right|_{W^{1,1}\left(\Omega^{h}\right)} \leq J_{h}\left(v^{h}\right) \leq C_{2}\left|v^{h}\right|_{W^{1,1}\left(\Omega^{h}\right)} .
$$


For some intermediate estimates we need second-order continuous and discrete seminorms, so we define for $v$ in the Sobolev space $W^{2,1}(2 \Omega)$ with periodic boundary conditions (i.e., treating $2 \Omega$ as a torus)

$$
|v|_{W^{2,1}(2 \Omega)}=\int_{2 \Omega}\left|D_{1}^{2} v\right|+\left|D_{2}^{2} v\right|
$$

and for periodic discrete functions $v^{h}$ on $2 \Omega^{h}$

$$
\begin{aligned}
&\left|v^{h}\right|_{W_{h}^{2,1}\left(2 \Omega^{h}\right)}=\sum_{i \in 2 \Omega^{h}}\left\{\left|\frac{v_{i+(1,0)}^{h}-2 v_{i}^{h}+v_{i-(1,0)}^{h}}{h^{2}}\right|\right. \\
&\left.+\left|\frac{v_{i+(0,1)}^{h}-2 v_{i}^{h}+v_{i-(0,1)}^{h}}{h^{2}}\right|\right\} h^{2} .
\end{aligned}
$$

Note that these seminorms do not include "cross" derivatives or differences, but we do not need these in our estimates.

Lemma 1.4 (TV difference). For any two discrete functionals $J_{\oplus \ominus}$ and $J_{\oplus^{\prime} \ominus^{\prime}}$, and any discrete function $v^{h}$ we have

$$
\left|J_{\oplus \ominus}\left(v^{h}\right)-J_{\oplus^{\prime} \ominus^{\prime}}\left(v^{h}\right)\right| \leq h\left|\operatorname{Ext}_{h} v^{h}\right|_{W_{h}^{2,1}\left(2 \Omega^{h}\right)} .
$$

Proof. First we consider $\left|J_{++}\left(v^{h}\right)-J_{+-}\left(v^{h}\right)\right|$. The quantities summed in (1.8) are the norms of two-vectors of divided differences, which we choose to write in the following way.

$$
\begin{aligned}
& \left|J_{++}\left(v^{h}\right)-J_{+-}\left(v^{h}\right)\right| \\
& =\left|\sum_{i \in \Omega^{h}}\right| \frac{1}{h}\left(\begin{array}{c}
\operatorname{Ext}_{h} v_{i+(1,0)}^{h}-\operatorname{Ext}_{h} v_{i}^{h} \\
\operatorname{Ext}_{h} v_{i+(0,1)}^{h}-\operatorname{Ext}_{h} v_{i}^{h}
\end{array}\right) \mid h^{2} \\
& -\sum_{i \in \Omega^{h}}\left|\frac{1}{h}\left(\begin{array}{c}
\operatorname{Ext}_{h} v_{i+(1,0)}^{h}-\operatorname{Ext}_{h} v_{i}^{h} \\
(-1)\left(\operatorname{Ext}_{h} v_{i-(0,1)}^{h}-\operatorname{Ext}_{h} v_{i}^{h}\right)
\end{array}\right)\right| h^{2} \mid \\
& \leq \frac{1}{h} \sum_{i \in \Omega^{h}}\left\|\left(\begin{array}{c}
\operatorname{Ext}_{h} v_{i+(1,0)}^{h}-\operatorname{Ext}_{h} v_{i}^{h} \\
\operatorname{Ext}_{h} v_{i+(0,1)}^{h}-\operatorname{Ext}_{h} v_{i}^{h}
\end{array}\right)|-|\left(\begin{array}{c}
\operatorname{Ext}_{h} v_{i+(1,0)}^{h}-\operatorname{Ext}_{h} v_{i}^{h} \\
(-1)\left(\operatorname{Ext}_{h} v_{i-(0,1)}^{h}-\operatorname{Ext}_{h} v_{i}^{h}\right)
\end{array}\right)\right\| h^{2} \\
& \leq \frac{1}{h} \sum_{i \in \Omega^{h}}\left|\left(\begin{array}{c}
\operatorname{Ext}_{h} v_{i+(1,0)}^{h}-\operatorname{Ext}_{h} v_{i}^{h} \\
\operatorname{Ext}_{h} v_{i+(0,1)}^{h}-\operatorname{Ext}_{h} v_{i}^{h}
\end{array}\right)-\left(\begin{array}{c}
\operatorname{Ext}_{h} v_{i+(1,0)}^{h}-\operatorname{Ext}_{h} v_{i}^{h} \\
(-1)\left(\operatorname{Ext}_{h} v_{i-(0,1)}^{h}-\operatorname{Ext}_{h} v_{i}^{h}\right)
\end{array}\right)\right| h^{2} \\
& =\frac{1}{h} \sum_{i \in \Omega^{h}}\left|\left(\operatorname{Ext}_{h} v_{i+(0,1)}^{h}-2 \operatorname{Ext}_{h} v_{i}^{h}+\operatorname{Ext}_{h} v_{i-(0,1)}^{h}\right)\right| h^{2} \\
& \leq h\left|\operatorname{Ext}_{h} v^{h}\right|_{W_{h}^{2,1}\left(2 \Omega^{h}\right)} \text {. }
\end{aligned}
$$

Identical arguments work to bound $\left|J_{++}\left(v^{h}\right)-J_{-+}\left(v^{h}\right)\right|,\left|J_{--}\left(v^{h}\right)-J_{+-}\left(v^{h}\right)\right|$, and 
$\left|J_{--}\left(v^{h}\right)-J_{-+}\left(v^{h}\right)\right|$. Finally we have

$$
\begin{aligned}
\left|J_{++}\left(v^{h}\right)-J_{--}\left(v^{h}\right)\right| & \leq \frac{1}{h} \sum_{i \in \Omega^{h}}\left|\left(\begin{array}{c}
\operatorname{Ext}_{h} v_{i+(1,0)}^{h}-2 \operatorname{Ext}_{h} v_{i}^{h}+\operatorname{Ext}_{h} v_{i-(1,0)}^{h} \\
\operatorname{Ext}_{h} v_{i+(0,1)}^{h}-2 \operatorname{Ext}_{h} v_{i}^{h}+\operatorname{Ext}_{h} v_{i-(0,1)}^{h}
\end{array}\right)\right| h^{2} \\
\leq & \frac{1}{h} \sum_{i \in \Omega^{h}}\left\{\left|\left(\begin{array}{c}
0 \\
\operatorname{Ext}_{h} v_{i+(0,1)}^{h}-2 \operatorname{Ext}_{h} v_{i}^{h}+\operatorname{Ext}_{h} v_{i-(0,1)}^{h}
\end{array}\right)\right|\right. \\
& \left.\quad||\left(\begin{array}{c}
\operatorname{Ext}_{h} v_{i+(1,0)}^{h}-2 \operatorname{Ext}_{h} v_{i}^{h}+\operatorname{Ext}_{h} v_{i-(1,0)}^{h} \\
0
\end{array}\right) \mid\right\} h^{2} \\
= & h\left|\operatorname{Ext}_{h} v^{h}\right|_{W_{h}^{2,1}\left(2 \Omega^{h}\right)} .
\end{aligned}
$$

1.3. Projectors, injectors, and smoothing operators. We define the piecewise constant injector of discrete functions $v^{h}$ into $L^{p}(\Omega)$ :

$$
\left(I_{h} v^{h}\right)(x)=v_{i}^{h} \quad \text { for } x \in \Omega_{i},
$$

where

$$
\Omega_{i}:=h(\Omega+i)
$$

Later we define an injector into a space of continuous, piecewise linear functions.

We also consider the piecewise constant projector of $v \in L^{1}(\Omega)$ onto the space of discrete functions, defined by

$$
\left(P_{h} v\right)_{i}=\frac{1}{\left|\Omega_{i}\right|} \int_{\Omega_{i}} v, \quad i \in \Omega^{h},
$$

where $\left|\Omega_{i}\right|$ is the measure of $\Omega_{i}$.

With these definitions, we collect some well-known results into the following lemma.

Lemma 1.5 (Injector and projector). There exists a constant $C$ such that for all $v \in L^{p}(\Omega)$ and $v^{h} \in L^{p}\left(\Omega^{h}\right), 1 \leq p \leq \infty$, the following properties hold.

For the projector $P_{h}$

$$
\left\|P_{h} v\right\|_{L^{p}\left(\Omega^{h}\right)} \leq\|v\|_{L^{p}(\Omega)} ;
$$

and

$$
\omega\left(P_{h} v, m\right)_{L^{p}\left(\Omega^{h}\right)} \leq C \omega(v, m h)_{L^{p}(\Omega)} .
$$

For the injector $I_{h}$

$$
\left\|v^{h}\right\|_{L^{p}\left(\Omega^{h}\right)}=\left\|I_{h} v^{h}\right\|_{L^{p}(\Omega)}
$$

and

$$
\omega\left(I_{h} v^{h}, m h\right)_{L^{p}(\Omega)} \leq C \omega\left(v^{h}, m\right)_{L^{p}\left(\Omega^{h}\right)} .
$$

We also have

$$
\left\|v-I_{h} P_{h} v\right\|_{L^{p}(\Omega)} \leq C \omega(v, h)_{L^{p}(\Omega)} .
$$


Finally, we have for any periodic $v \in W^{2,1}(2 \Omega)$

$$
\left|P_{h} v\right|_{W_{h}^{2,1}\left(2 \Omega^{h}\right)} \leq|v|_{W^{2,1}(2 \Omega)} .
$$

Proof. The relation (1.22) follows from definitions. Jensen's inequality implies (1.20) and (1.21). Property (1.23) follows from the definitions of discrete and continuous modulus of continuity and the fact that for any $1 \leq p \leq \infty$ and $\tau>1$ there exists a $C$ such that for all $t>0$

$$
\omega(f, \tau t)_{L^{p}(\Omega)} \leq C \omega(f, t)_{L^{p}(\Omega)} .
$$

We note (1.24) is a special case of a general bound for the error in spline approximation; see [8], Theorem 7.3, page 225 .

To prove (1.25), we deal with the differences in the horizontal direction.

$$
\begin{aligned}
& \sum_{i \in 2 \Omega^{h}}\left|\frac{\left(P_{h} v\right)_{i+(1,0)}-2\left(P_{h} v\right)_{i}+\left(P_{h}\right) v_{i-(1,0)}}{h^{2}}\right| h^{2} \\
= & \sum_{i \in 2 \Omega^{h}} \frac{1}{h}\left|\frac{\left(P_{h} v\right)_{i+(1,0)}-\left(P_{h} v\right)_{i}}{h}-\frac{\left(P_{h} v\right)_{i}-\left(P_{h} v\right)_{i-(1,0)}}{h}\right| h^{2} \\
= & \sum_{i \in 2 \Omega^{h}} \frac{1}{h^{2}}\left|\int_{\Omega_{i}}[(v(x+h, y)-v(x, y))-(v(x, y)-v(x-h, y))] d x d y\right| \\
= & \sum_{i \in 2 \Omega^{h}} \frac{1}{h^{2}}\left|\int_{\Omega_{i}} \int_{0}^{h}\left[D_{1} v(x+t, y)-D_{1} v(x+t-h, y)\right] d t d x d y\right| \\
= & \sum_{i \in 2 \Omega} \frac{1}{h^{2}}\left|\int_{\Omega_{i}} \int_{0}^{h} \int_{-h}^{0} D_{11} v(x+t+s, y) d s d t d x d y\right| \\
\leq & \left.\int_{2 \Omega}\left|D_{11} v\right| d x d y \quad \text { (exchange the order of integration and sum over } i\right)
\end{aligned}
$$

Arguing similarly in the vertical direction, we see that (1.25) holds. $\square$

We have need of another map taking $v^{h} \in L^{2}\left(\Omega^{h}\right)$ to $L^{2}(\Omega)$, in the form of a piecewise linear interpolant of the discrete values of $v_{i}^{h}$. To this end, let $\phi$ be the box spline function whose support is the hexagon $D$ in Figure 1.1 with $\phi$ being linear on each triangle in Figure 1.1 and

$$
\phi(i)= \begin{cases}1, & i=(0,0), \\ 0, & i \neq(0,0), \quad i \in \mathbb{Z}^{2} .\end{cases}
$$

We dilate and translate $\phi$ to obtain the function

$$
\phi_{i}^{h}(x):=\phi\left(\frac{x}{h}-\left(i+\left(\frac{1}{2}, \frac{1}{2}\right)\right)\right) .
$$

We see that $\operatorname{supp} \phi_{i}^{h}$ is $D$ dilated by $h$ and translated by $\left(i+\left(\frac{1}{2}, \frac{1}{2}\right)\right) h$.

We define the interpolant Int $v^{h}$ by

$$
\operatorname{Int} v^{h}=\sum_{i \in \mathbb{Z}^{2}} \operatorname{Ext}_{h} v_{i}^{h} \phi_{i} .
$$




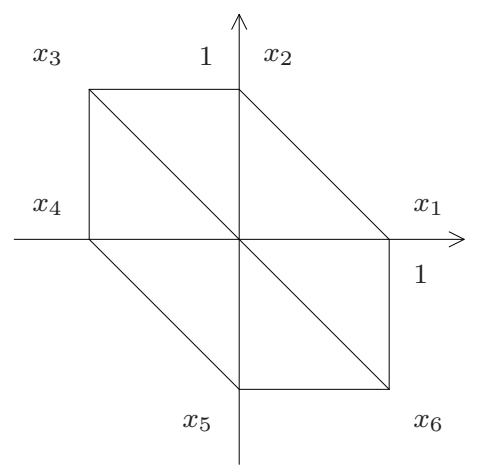

FIG. 1.1. D, the support of $\phi$

We then have the following lemma.

Lemma 1.6 (Piecewise linear injector). For any $v^{h}$ be in $L^{2}\left(\Omega^{h}\right)$ we have

$$
\left|\operatorname{Int} v^{h}\right|_{\mathrm{BV}(\Omega)}=\frac{1}{2}\left(J_{++}\left(v^{h}\right)+J_{--}\left(v^{h}\right)\right) .
$$

Additionally, there exists a constant $C$ such that for all discrete functions $v^{h}$

$$
\left\|I_{h} v^{h}-\operatorname{Int} v^{h}\right\|_{L^{2}(\Omega)} \leq C \omega\left(v^{h}, 1\right)_{L^{2}\left(\Omega^{h}\right)} .
$$

Proof. The proof of (1.27) is just a calculation, which we leave to the reader. (The $J_{++}$terms come from triangles with the orientation of the triangle in the upper-right quadrant of Figure 1.1, and the $J_{--}$terms come from triangles with the orientation of the triangle in the lower-left quadrant of Figure 1.1.)

To prove (1.28), we consider each subsquare $\Omega_{i}$, where $I_{h} v^{h}$ takes the value $v_{i}^{h}$. The definition of $\Omega_{i}$ is in (1.19). We construct the constant function

$$
w^{h}=\sum_{j^{\prime} \in \Omega^{h}} v_{i}^{h} \phi_{j^{\prime}}^{h}
$$

which equals $I_{h} v^{h}$ on $\Omega_{i}$, and we set

$$
I^{\prime}=\left\{i^{\prime} \mid \operatorname{supp} \phi_{i^{\prime}}^{h} \text { intersects } \Omega_{i} \text { nontrivially }\right\} .
$$

There are precisely seven elements in $I^{\prime}$. Then

$$
\begin{aligned}
\int_{\Omega_{i}}\left|\operatorname{Int} v^{h}-I_{h} v^{h}\right|^{2} & =\int_{\Omega_{i}}\left|\sum_{i^{\prime} \in I^{\prime}}\left(v_{i}^{h}-v_{i^{\prime}}^{h}\right) \phi_{i^{\prime}}\right|^{2} \\
& \leq \int_{\Omega_{i}}\left|\sum_{i^{\prime} \in I^{\prime}}\right| v_{i}^{h}-v_{i^{\prime}}^{h}||^{2} \text { since }\left|\phi_{i^{\prime}}^{h}(x)\right| \leq 1 \\
& \leq C \sum_{i^{\prime} \in I^{\prime}}\left|v_{i}^{h}-v_{i^{\prime}}^{h}\right|^{2} h^{2}
\end{aligned}
$$

For one of the "diagonal" terms we have

$$
\left|v_{i+(-1,1)}^{h}-v_{i}^{h}\right|^{2} \leq 2\left(\left|v_{i+(-1,1)}^{h}-v_{i+(-1,0)}^{h}\right|^{2}+\left|v_{i+(-1,0)}^{h}-v_{i}^{h}\right|^{2}\right) ;
$$


a similar bound exists for $\left|v_{i+(1,-1)}^{h}-v_{i}^{h}\right|^{2}$.

Thus, there exists a $C$ such that

$$
\begin{aligned}
\int_{\Omega}\left|\operatorname{Int} v^{h}-I_{h} v^{h}\right|^{2} & =\sum_{i \in \Omega^{h}} \int_{\Omega_{i}}\left|\operatorname{Int} v^{h}-I_{h} v^{h}\right|^{2} \\
& \leq C \sum_{i \in \Omega^{h}}\left\{\left|v_{i+(1,0)}^{h}-v_{i}^{h}\right|^{2}+\left|v_{i+(0,1)}^{h}-v_{i}^{h}\right|^{2}\right\} h^{2}
\end{aligned}
$$

so there exists another constant $C$ such that

$$
\begin{aligned}
\left\|\operatorname{Int} v^{h}-I_{h} v^{h}\right\|_{L^{2}(\Omega)} & \leq C\left(\left\|T_{(0,1)} v^{h}-v^{h}\right\|_{L^{2}\left(\Omega^{h}\right)}+\left\|T_{(1,0)} v^{h}-v^{h}\right\|_{L^{2}\left(\Omega^{h}\right)}\right) \\
& \leq C \omega\left(v^{h}, 1\right)_{L^{2}\left(\Omega^{h}\right)}
\end{aligned}
$$

We need both continuous and discrete smoothing operators, which we define as follows. Assume that $\eta(x)$ is a a fixed nonnegative, rotationally symmetric, function with support in the unit disk; further, suppose that $\eta$ is $C^{\infty}$ and has integral 1. For $\epsilon>0$ we define the scaled function

$$
\eta_{\epsilon}(x):=\frac{1}{\epsilon^{2}} \eta\left(\frac{x}{\epsilon}\right), \quad x \in \mathbb{R}^{2} ;
$$

we smooth a function $v \in L^{p}(\Omega), 1 \leq p \leq \infty$, by computing

$$
\left(\mathcal{S}_{\epsilon} v\right)(x):=\left(\eta_{\epsilon} * \operatorname{Ext} v\right)(x)=\int_{\mathbb{R}^{2}} \eta_{\epsilon}(x-y) \operatorname{Ext} v(y) d y, \quad x \in \mathbb{R}^{2} .
$$

Our discrete smoothing operator is defined simply as

$$
S_{L} v^{h}:=\frac{1}{(2 L+1)^{2}} \sum_{|\ell| \leq L} T_{\ell} \operatorname{Ext}_{h} v^{h} .
$$

It's clear from these definitions that

$$
T_{\ell} S_{L} \operatorname{Ext}_{h} v^{h}=S_{L} T_{\ell} \operatorname{Ext}_{h} v^{h} \text { and } \mathcal{T}_{\tau} \mathcal{S}_{\epsilon} \operatorname{Ext} v=\mathcal{S}_{\epsilon} \mathcal{T}_{\tau} \operatorname{Ext} v
$$

and that for any $1 \leq p \leq \infty$

$$
\left\|S_{L} v^{h}\right\|_{L^{p}\left(\Omega^{h}\right)} \leq\left\|S_{L} v^{h}\right\|_{L^{p}\left(2 \Omega^{h}\right)} \leq\left\|\operatorname{Ext}_{h} v^{h}\right\|_{L^{p}\left(2 \Omega^{h}\right)}=4\left\|v^{h}\right\|_{L^{p}\left(\Omega^{h}\right)}
$$

and

$$
\left\|\mathcal{S}_{\epsilon} v\right\|_{L^{p}(\Omega)} \leq\left\|\mathcal{S}_{\epsilon} v\right\|_{L^{p}(2 \Omega)} \leq\|\operatorname{Ext} v\|_{L^{p}(2 \Omega)}=4\|v\|_{L^{p}(\Omega)} .
$$

For these continuous and discrete smoothing operators we have the following results.

Lemma 1.7 (Smoothing operators). There exists a constant $C>0$ such that the following inequalities hold.

For all $v \in L^{p}(\Omega), p \geq 1$, and all discrete functions $v^{h}$, we have

$$
J_{*}\left(S_{L} v^{h}\right) \leq J_{*}\left(v^{h}\right)
$$


and

$$
\left|\mathcal{S}_{\epsilon} v\right|_{\mathrm{BV}(\Omega)} \leq|v|_{\mathrm{BV}(\Omega)} .
$$

Similarly, for all $M, t>0$,

$$
\omega\left(S_{L} v^{h}, M\right)_{L^{2}\left(\Omega^{h}\right)} \leq C \omega\left(v^{h}, M\right)_{L^{2}\left(\Omega^{h}\right)}
$$

and

$$
\omega\left(\mathcal{S}_{\epsilon} v, t\right)_{L^{2}(\Omega)} \leq C \omega(v, t)_{L^{2}(\Omega)} .
$$

Furthermore,

$$
\left\|S_{L} v^{h}-v^{h}\right\|_{L^{2}\left(\Omega^{h}\right)} \leq C \omega\left(v^{h}, L\right)_{L^{2}\left(\Omega^{h}\right)},
$$

and

$$
\left\|\mathcal{S}_{\epsilon} v-v\right\|_{L^{2}(\Omega)} \leq C \omega(v, \epsilon)_{L^{2}(\Omega)} .
$$

We also have

$$
\left|\mathcal{S}_{\epsilon} v\right|_{W^{2,1}(2 \Omega)} \leq \frac{C}{\epsilon}|v|_{\mathrm{BV}(\Omega)}
$$

and

$$
\left|S_{L} v^{h}\right|_{W_{h}^{2,1}\left(2 \Omega^{h}\right)} \leq \frac{C}{L h}\left|v^{h}\right|_{W^{1,1}\left(\Omega^{h}\right)} .
$$

Proof. Using the notation and results of Lemma 1.2, because $J_{*}$ is convex, we have

$$
\begin{aligned}
J_{*}\left(S_{L} v^{h}\right)=J_{*}^{\Omega^{h}}\left(S_{L} v^{h}\right) & =\frac{1}{4} J_{*}^{2 \Omega^{h}}\left(S_{L} v^{h}\right) \\
& =\frac{1}{4} J_{*}^{2 \Omega^{h}}\left(\frac{1}{(2 L+1)^{2}} \sum_{|\ell| \leq L} T_{\ell} \operatorname{Ext}_{h} v^{h}\right) \\
& \leq \frac{1}{4} \frac{1}{(2 L+1)^{2}} \sum_{|\ell| \leq L} J_{*}^{2 \Omega^{h}}\left(T_{\ell} \operatorname{Ext}_{h} v^{h}\right) \\
& =\frac{1}{4} J_{*}^{2 \Omega^{h}}\left(\operatorname{Ext}_{h} v^{h}\right) \\
& =J_{*}^{\Omega^{h}}\left(v^{h}\right) .
\end{aligned}
$$

For the next inequality, we have

$$
\left|\mathcal{S}_{\epsilon} v\right|_{\mathrm{BV}(\Omega)}=\frac{1}{4}\left|\mathcal{S}_{\epsilon} v\right|_{\mathrm{BV}(2 \Omega)} \leq \frac{1}{4}|v|_{\mathrm{BV}(2 \Omega)}=|v|_{\mathrm{BV}(\Omega)},
$$

since it is clear from the definition of $|v|_{\mathrm{BV}(2 \Omega)}$ with periodic boundary conditions that $\left|\mathcal{S}_{\epsilon} v\right|_{\mathrm{BV}(2 \Omega)} \leq|v|_{\mathrm{BV}(2 \Omega)}$ (throw the mollifier on $\phi$ ).

The two inequalities (1.34) and (1.35) follow from the definitions of $S_{L}$ and $\mathcal{S}_{\epsilon}$ and Lemma 1.1. 
We have

$$
\begin{aligned}
\left\|S_{L} v^{h}-v^{h}\right\|_{L^{2}\left(\Omega^{h}\right)} & \leq\left\|S_{L} v^{h}-\operatorname{Ext}_{h} v^{h}\right\|_{L^{2}\left(2 \Omega^{h}\right)} \\
& =\frac{1}{(2 L+1)^{2}}\left\|\sum_{|\ell| \leq L}\left(T_{\ell} \operatorname{Ext}_{h} v^{h}-\operatorname{Ext}_{h} v^{h}\right)\right\|_{L^{2}\left(2 \Omega^{h}\right)} \\
& \leq \frac{1}{(2 L+1)^{2}} \sum_{|\ell| \leq L}\left\|T_{\ell} \operatorname{Ext}_{h} v^{h}-\operatorname{Ext}_{h} v^{h}\right\|_{L^{2}\left(2 \Omega^{h}\right)} \\
& \leq \omega\left(\operatorname{Ext}_{h} v^{h}, L\right)_{L^{2}\left(2 \Omega^{h}\right)} \\
& \leq C \omega\left(v^{h}, L\right)_{L^{2}\left(\Omega^{h}\right)}
\end{aligned}
$$

Similarly, since the support of $\eta_{\epsilon}(x)$ is contained in the ball $|x| \leq \epsilon$,

$$
\begin{aligned}
\left\|\mathcal{S}_{\epsilon} v-v\right\|_{L^{2}(\Omega)} & \leq\left\|\mathcal{S}_{\epsilon} v-\operatorname{Ext} v\right\|_{L^{2}(2 \Omega)} \\
& =\left\|\eta_{\epsilon} * \operatorname{Ext} v-\operatorname{Ext} v\right\|_{L^{2}(2 \Omega)} \\
& \leq \int_{2 \Omega} \eta_{\epsilon}(y)\|\operatorname{Ext} v(\cdot-y)-\operatorname{Ext} v\|_{L^{2}(2 \Omega)} d y \\
& \leq C \omega(v, \epsilon)_{L^{2}(\Omega)} .
\end{aligned}
$$

The last line follows from (1.4) of Lemma 1.1.

The bound on the discrete $W_{h}^{2,1}\left(2 \Omega^{h}\right)$ semi-norm is a typical inverse inequality; to deal with the differences in the horizontal direction,

$$
\begin{aligned}
& \sum_{i \in 2 \Omega^{h}}\left|\frac{S_{L} v_{i+(1,0)}^{h}-2 S_{L} v_{i}^{h}+S_{L} v_{i-(1,0)}^{h}}{h^{2}}\right| h^{2} \\
= & \sum_{i \in 2 \Omega^{h}}\left|\frac{1}{(2 L+1)^{2}} \sum_{|\ell| \leq L} \frac{\operatorname{Ext}_{h} v_{i+(1,0)+\ell}^{h}-2 \operatorname{Ext}_{h} v_{i+\ell}^{h}+\operatorname{Ext}_{h} v_{i+\ell-(1,0)}^{h}}{h^{2}}\right| h^{2} \\
= & \sum_{i \in 2 \Omega^{h}} \frac{1}{(2 L+1)^{2}} \mid \sum_{\left|\ell_{2}\right| \leq L} \frac{\operatorname{Ext}_{h} v_{i+\left(L+1, \ell_{2}\right)}^{h}-\operatorname{Ext}_{h} v_{i+\left(L, \ell_{2}\right)}^{h}}{h^{2}} \\
\leq & \sum_{i \in 2 \Omega^{h}} \frac{1}{(2 L+1)^{2}} \sum_{\left|\ell_{2}\right| \leq L}\left\{\frac{\operatorname{Ext}_{h} v_{i-\left(L, \ell_{2}\right)}^{h}-\operatorname{Ext}_{h} v_{i-\left(L+1, \ell_{2}\right)}^{h}}{h^{2}} \mid h^{2} \quad \text { (sum over } \ell_{1}\right) \\
h^{2} & \left.+\left|\frac{\operatorname{Ext}_{h} v_{i-\left(L, \ell_{2}\right)}^{h}-\operatorname{Ext}_{h} v_{i-\left(L+1, \ell_{2}\right)}^{h}}{h^{2}}\right|\right\} h^{2} \\
\leq & \frac{C}{(2 L+1) h} \sum_{i \in 2 \Omega^{h}}\left|\frac{\operatorname{Ext}_{h} v_{i+(1,0)}^{h}-\operatorname{Ext}_{h} v_{i}^{h}}{h}\right| h^{2} \\
\leq & \frac{C}{L h}\left|v^{h}\right|_{W^{1,1}\left(\Omega^{h}\right)}
\end{aligned}
$$

For the bound on the $W^{2,1}(2 \Omega)$ semi-norm, again we deal with derivatives in only one direction. We prove 


$$
\int_{2 \Omega}\left|D_{1}^{2} \mathcal{S}_{\epsilon} v\right| \leq \frac{C}{\epsilon} \int_{\Omega}|D v|
$$

In fact

$$
\begin{aligned}
\int_{2 \Omega}\left|D_{1}^{2} \mathcal{S}_{\epsilon} v\right| & =\sup _{\phi \in C_{0}^{1}(2 \Omega),|\phi| \leq 1} \int_{\mathbb{R}^{2}}\left(D_{1}^{2} \mathcal{S}_{\epsilon} v\right) \phi \\
& =\sup _{\phi \in C_{0}^{1}(2 \Omega),|\phi| \leq 1} \int_{\mathbb{R}^{2}}\left(D_{1} \mathcal{S}_{\epsilon} v\right) D_{1}(-\phi) \\
& =\sup _{\phi \in C_{0}^{1}(2 \Omega),|\phi| \leq 1} \int_{\mathbb{R}^{2}} D_{1}\left(\eta_{\epsilon} * \operatorname{Ext} v\right) D_{1}(-\phi) \\
& =\sup _{\phi \in C_{0}^{1}(2 \Omega),|\phi| \leq 1} \int_{\mathbb{R}^{2}}(\operatorname{Ext} v) D_{1}\left(D_{1} \eta_{\epsilon} * \phi\right) ;
\end{aligned}
$$

note that all but the first of these integrals are over $\mathbb{R}^{2}$. Notice

$$
\left|D_{1} \eta_{\epsilon} * \phi\right| \leq\left\|D_{1} \eta_{\epsilon}\right\|_{L^{1}}\|\phi\|_{\infty} \leq \frac{C}{\epsilon}
$$

and $D_{1} \eta_{\epsilon} * \phi \in C_{0}^{\infty}\left(2 \Omega^{\epsilon}\right)$, where

$$
\Omega^{\epsilon}:=\{x \mid \operatorname{dist}(x, 2 \bar{\Omega}) \leq \epsilon\},
$$

therefore

$$
\begin{aligned}
\int_{2 \Omega}\left|D_{1}^{2} \mathcal{S}_{\epsilon} v\right| & \leq \frac{C}{\epsilon} \int_{2 \Omega^{\epsilon}}|D \operatorname{Ext} v| \\
& \leq \frac{C}{\epsilon} \int_{\Omega^{\prime}}|D \operatorname{Ext} v| \leq \frac{C}{\epsilon} \int_{\Omega}|D v| .
\end{aligned}
$$

where $\Omega^{\prime}=\{(x, y)|| x|| y \mid, \leq 3\}$.

The same result holds for $\int_{\Omega}\left|D_{2}^{2} \mathcal{S}_{\epsilon} v\right|$.

2. Relationships between discrete and continuous variation and functionals. We need to compare continuous and discrete variation functionals, so we have the following lemma.

Lemma 2.1 (TV bound). There exists a $C>0$ such for any $J_{h}$ and any $v \in$ $L^{1}(\Omega)$

$$
J_{h}\left(P_{h} v\right) \leq|v|_{\mathrm{BV}(\Omega)}+C h|\operatorname{Ext} v|_{W^{2,1}(2 \Omega)}
$$

and for any $v^{h}$ defined on $\Omega^{h}$

$$
\left|\operatorname{Int} v^{h}\right|_{\mathrm{BV}(\Omega)} \leq J_{h}\left(v^{h}\right)+C h\left|\operatorname{Ext}_{h} v^{h}\right|_{W_{h}^{2,1}\left(2 \Omega^{h}\right)} .
$$

Proof. One proves the second inequality simply by combining (1.27) and (1.18).

As for the first inequality, the left hand side is finite for $v \in L^{1}(\Omega)$, so if $\operatorname{Ext} v \notin$ $W^{2,1}(2 \Omega)$, we're done. So we assume that Ext $v \in W^{2,1}(2 \Omega)$ and we prove (2.1) for $J_{h}=J_{++}$, the other cases being the same. 
We denote $P_{h} v$ by $v^{h}$ and write

$$
\nabla_{x}^{+} v_{i}^{h}=\frac{\operatorname{Ext}_{h} v_{i+(1,0)}^{h}-\operatorname{Ext}_{h} v_{i}^{h}}{h}, \quad \nabla_{y}^{+} v_{i}^{h}=\frac{\operatorname{Ext}_{h} v_{i+(0,1)}^{h}-\operatorname{Ext}_{h} v_{i}^{h}}{h} .
$$

We briefly note that when $i \in \Omega^{h}$ then $\operatorname{Ext}_{h} v_{i}^{h}=v_{i}^{h}$ and these differences are zero if $i+(0,1)$ or $i+(1,0)$ are outside $\Omega^{h}$. In the argument that follows, we write $v$ for Ext $v$.

Then

$$
\begin{aligned}
\nabla_{x}^{+} v_{i}^{h}-\frac{1}{h^{2}} \int_{\Omega_{i}} D_{1} v & =\frac{v_{i+(1,0)}^{h}-v_{i}^{h}}{h}-\frac{1}{h^{2}} \int_{\Omega_{i}} D_{1} v \\
& =\frac{1}{h} \frac{1}{\left|\Omega_{i}\right|} \int_{\Omega_{i}}[v(x+h, y)-v(x, y)] d x d y-\frac{1}{h^{2}} \int_{\Omega_{i}} D_{1} v .
\end{aligned}
$$

The integrand of the first integral can be rewritten as an integral of $D_{1} v$, then combining these two integrals and once again rewriting the integrand as an integral of the second derivative of $v$, we have

$$
\begin{aligned}
\nabla_{x}^{+} v_{i}^{h}-\frac{1}{h^{2}} \int_{\Omega_{i}} D_{1} v & =\frac{1}{h^{3}} \int_{\Omega_{i}}\left(\int_{0}^{h} D_{1} v(x+t, y) d t-h D_{1} v(x, y)\right) d x d y \\
& =\frac{1}{h^{3}} \int_{\Omega_{i}} \int_{0}^{h}\left(D_{1} v(x+t, y)-D_{1} v(x, y)\right) d t d x d y \\
& =\frac{1}{h^{3}} \int_{\Omega_{i}} \int_{0}^{h} \int_{0}^{t} D_{1}^{2} v(x+s, y) d s d t d x d y
\end{aligned}
$$

Therefore

$$
\nabla_{x}^{+} v_{i}^{h}=\frac{1}{h^{2}} \int_{\Omega_{i}} D_{1} v+\frac{1}{h^{3}} \int_{\Omega_{i}} \int_{0}^{h} \int_{0}^{t} D_{1}^{2} v(x+s, y) d s d t d x d y .
$$

Similarly,

$$
\nabla_{y}^{+} v_{i}^{h}=\frac{1}{h^{2}} \int_{\Omega_{i}} D_{2} v+\frac{1}{h^{3}} \int_{\Omega_{i}} \int_{0}^{h} \int_{0}^{t} D_{2}^{2} v(x, y+s) d s d t d x d y .
$$

So we can bound the norm of $\nabla^{+} v_{i}^{h}$ by

$$
\begin{aligned}
\left|\nabla^{+} v_{i}^{h}\right| \leq & \frac{1}{h^{2}}\left|\left(\begin{array}{c}
\int_{\Omega_{i}} D_{1} v \\
\int_{\Omega_{i}} D_{2} v
\end{array}\right)\right| \\
& +\frac{1}{h^{3}}\left|\left(\begin{array}{c}
\int_{\Omega_{i}} \int_{0}^{h} \int_{0}^{t} D_{1}^{2} v(x+s, y) d s d t d x d y \\
\int_{\Omega_{i}} \int_{0}^{h} \int_{0}^{t} D_{2}^{2} v(x, y+s) d s d t d x d y
\end{array}\right)\right| \\
\leq & \frac{1}{h^{2}} \int_{\Omega_{i}}|D v| \\
& +\frac{1}{h^{3}} \int_{\Omega_{i}} \int_{0}^{h} \int_{0}^{t}\left|D_{1}^{2} v(x+s, y)\right| d s d t d x d y \\
& +\frac{1}{h^{3}} \int_{\Omega_{i}} \int_{0}^{h} \int_{0}^{t}\left|D_{2}^{2} v(x, y+s)\right| d s d t d x d y
\end{aligned}
$$


The last line follows from the fact that

$$
\left|\left(\begin{array}{c}
\int f \\
\int g
\end{array}\right)\right| \leq \int \sqrt{f^{2}+g^{2}}
$$

by Jensen's inequality, and

$$
\left|\left(\begin{array}{c}
a \\
b
\end{array}\right)\right| \leq|a|+|b|
$$

To bound the discrete total variation $J_{++}\left(v^{h}\right)$, we sum (2.4) over all indices $i \in \Omega^{h}$ with weight $h^{2}$ at each index. We obtain

$$
J_{++}\left(v^{h}\right) \leq \int_{\Omega}|D v|+e_{1}+e_{2}
$$

where

$$
\begin{aligned}
e_{1} & =\sum_{i} h^{2} \frac{1}{h^{3}} \int_{\Omega_{i}} \int_{0}^{h} \int_{0}^{t}\left|D_{1}^{2} v(x+s, y)\right| d s d t d x d y \\
& \leq \frac{1}{h} \int_{0}^{h} \int_{0}^{t}\left\{\int_{\Omega}\left|D_{1}^{2} v(x+s, y)\right| d x d y\right\} d s d t \\
& \leq \frac{C}{h} \int_{0}^{h} \int_{0}^{t}\left\{\int_{\Omega}\left|D_{1}^{2} v\right| d x d y\right\} d s d t \\
& \leq C h \int_{\Omega}\left|D_{1}^{2} v\right| .
\end{aligned}
$$

We also have

$$
\begin{aligned}
e_{2} & =\sum_{i} h^{2} \frac{1}{h^{3}} \int_{\Omega_{i}} \int_{0}^{h} \int_{0}^{t}\left|D_{2}^{2} v(x, y+s)\right| d s d t d x d y \\
& \leq C h \int_{\Omega}\left|D_{2}^{2} v\right| .
\end{aligned}
$$

Therefore

$$
J_{++}\left(v^{h}\right) \leq \int_{\Omega}|D v|+C h \int_{\Omega}\left(\left|D_{1}^{2} v\right|+\left|D_{2}^{2} v\right|\right) .
$$

By the same argument, we have the same bound for $J_{+-}, J_{-+}$, and $J_{--}$,

$$
J\left(v^{h}\right) \leq \int_{\Omega}|D v|+C h \int_{\Omega}\left(\left|D_{1}^{2} v\right|+\left|D_{2}^{2} v\right|\right),
$$

where $J \in\left\{J_{+-}, J_{-+}, J_{--}\right\}$. Thus, we complete the proof. $\square$

Our goal is to bound the difference between various continuous and discrete convex functionals defined on $L^{2}(\Omega)$ and $L^{2}\left(\Omega^{h}\right)$. We fix $\lambda>0$. Given $g \in L^{2}(\Omega)$, we consider the (unique) minimizer $u$ of the functional

$$
E(v)=\frac{1}{2 \lambda}\|v-g\|_{L^{2}(\Omega)}^{2}+|v|_{\mathrm{BV}(\Omega)}
$$


and the (unique) minimizer $u^{h}$ of the functional

$$
E_{h}\left(v^{h}\right)=\frac{1}{2 \lambda}\left\|v^{h}-P_{h} g\right\|_{L^{2}\left(\Omega^{h}\right)}^{2}+J_{h}\left(v^{h}\right),
$$

where $J_{h}$ is any of the discrete variational functionals defined above. Most of our analysis concerns itself with the special case

$$
E_{h}\left(v^{h}\right)=\frac{1}{2 \lambda}\left\|v^{h}-P_{h} g\right\|_{L^{2}\left(\Omega^{h}\right)}^{2}+J_{*}\left(v^{h}\right) .
$$

It is difficult to compare $u$ and $u^{h}$ directly, because $J_{*}\left(u^{h}\right)$ and $|u|_{\mathrm{BV}(\Omega)}$ could be far apart, in general, even if $u^{h} \rightarrow u$ as $h \rightarrow 0$. However, there are smoothed versions of $u$ and $u^{h}$, close to $u$ and $u^{h}$, whose continuous and discrete variations are close, as the following Lemma shows.

LEMma 2.2 (TV consistency). There exists a constant $C$ such that for any discrete function $v^{h} \in L^{2}\left(\Omega^{h}\right)$ and any positive integer $L$ we have

$$
\left|\operatorname{Int} S_{L} v^{h}\right|_{\mathrm{BV}(\Omega)} \leq J_{*}\left(v^{h}\right)+\frac{C}{L} J_{*}\left(v^{h}\right) .
$$

Furthermore, there is a constant $C$ such that for any $v \in \mathrm{BV}(\Omega)$ and any positive $\epsilon$ and any discrete functional $J_{h}$, we have

$$
J_{h}\left(P_{h} \mathcal{S}_{\epsilon} v\right) \leq|v|_{\mathrm{BV}(\Omega)}+\frac{C h}{\epsilon}|v|_{\mathrm{BV}(\Omega)} .
$$

Proof. For the first inequality, we have from (2.2) in Lemma 2.1 (with $J_{h}=J_{*}$ )

$$
\left|\operatorname{Int} S_{L} v^{h}\right|_{\mathrm{BV}(\Omega)} \leq J_{*}\left(S_{L} v^{h}\right)+C h\left|S_{L} v^{h}\right|_{W_{h}^{2,1}\left(2 \Omega^{h}\right)},
$$

while from (1.32) in Lemma $1.7 J_{*}\left(S_{L} v^{h}\right) \leq J_{*}\left(v^{h}\right)$ and (1.39) in the same lemma

$$
\left|S_{L} v^{h}\right|_{W_{h}^{2,1}\left(2 \Omega^{h}\right)} \leq \frac{C}{L h}\left|v^{h}\right|_{W^{1,1}\left(\Omega^{h}\right)} \leq \frac{C}{L h} J_{*}\left(v^{h}\right) .
$$

The second inequality follows from (1.16). Combining the previous inequalities gives (2.6).

For (2.7), we have from (2.1) in Lemma 2.1

$$
J_{h}\left(P_{h} \mathcal{S}_{\epsilon} v\right) \leq\left|\mathcal{S}_{\epsilon} v\right|_{\mathrm{BV}(\Omega)}+C h\left|\mathcal{S}_{\epsilon} v\right|_{W^{2,1}(2 \Omega)},
$$

while (1.33) yields

$$
\left|\mathcal{S}_{\epsilon} v\right|_{\operatorname{BV}(\Omega)} \leq|v|_{\operatorname{BV}(\Omega)}
$$

and (1.38) gives

$$
\left|\mathcal{S}_{\epsilon} v\right|_{W^{2,1}(2 \Omega)} \leq \frac{C}{\epsilon}|v|_{\mathrm{BV}(\Omega)} .
$$

Combining these three inequalities yields (2.7).

Now we compare discrete and continuous energy functionals. 
Lemma 2.3 (Comparing discrete and continuous energies). There exists a constant $C>0$ such that for all $J_{h}$ and for all $v \in \mathrm{BV}(\Omega)$

$$
\begin{aligned}
E_{h}\left(P_{h} \mathcal{S}_{\epsilon} v\right) & \leq E(v)+\frac{C h}{\epsilon}|v|_{\operatorname{BV}(\Omega)} \\
& +\frac{C}{\lambda}\|v-g\|_{L^{2}(\Omega)}\left(\omega(v, h)_{L^{2}(\Omega)}+\omega(v, \epsilon)_{L^{2}(\Omega)}+\omega(g, h)_{L^{2}(\Omega)}\right) \\
& +\frac{C}{\lambda}\left(\omega(v, h)_{L^{2}(\Omega)}^{2}+\omega(v, \epsilon)_{L^{2}(\Omega)}^{2}+\omega(g, h)_{L^{2}(\Omega)}^{2}\right) .
\end{aligned}
$$

Furthermore, if $J_{h}=J_{*}$, then for all discrete functions $v^{h}$

$$
\begin{aligned}
E\left(\operatorname{Int} S_{L} v^{h}\right) \leq & E_{h}\left(v^{h}\right)+\frac{C}{L} J_{*}\left(v^{h}\right) \\
& +\frac{C}{\lambda}\left\|v^{h}-P_{h} g\right\|_{L^{2}\left(\Omega^{h}\right)}\left(\omega\left(v^{h}, L\right)_{L^{2}\left(\Omega^{h}\right)}+\omega(g, h)_{L^{2}(\Omega)}\right) \\
& +\frac{C}{\lambda}\left(\omega\left(v^{h}, L\right)_{L^{2}(\Omega)}^{2}+\omega(g, h)_{L^{2}(\Omega)}^{2}\right) .
\end{aligned}
$$

Proof. We have

$$
E_{h}\left(P_{h} \mathcal{S}_{\epsilon} v\right)=J_{h}\left(P_{h} \mathcal{S}_{\epsilon} v\right)+\frac{1}{2 \lambda}\left\|P_{h} \mathcal{S}_{\epsilon} v-P_{h} g\right\|_{L^{2}\left(\Omega^{h}\right)}^{2} .
$$

From (2.7), we see that the first term on the right is bounded by

$$
|v|_{\mathrm{BV}(\Omega)}+\frac{C h}{\epsilon}|v|_{\mathrm{BV}(\Omega)} .
$$

Now

$$
\left\|P_{h} \mathcal{S}_{\epsilon} v-P_{h} g\right\|_{L^{2}\left(\Omega^{h}\right)}^{2}=\left\|I_{h} P_{h} \mathcal{S}_{\epsilon} v-I_{h} P_{h} g\right\|_{L^{2}(\Omega)}^{2},
$$

and the quantity on the right can be written as

$$
\begin{aligned}
& \left\|\left(I_{h} P_{h} \mathcal{S}_{\epsilon} v-\mathcal{S}_{\epsilon} v\right)+\left(\mathcal{S}_{\epsilon} v-v\right)+(v-g)+\left(g-I_{h} P_{h} g\right)\right\|_{L^{2}(\Omega)}^{2} \\
& \leq\|v-g\|_{L^{2}(\Omega)}^{2}+2\|v-g\|_{L^{2}(\Omega)} \\
& \quad \times\left\|\left(I_{h} P_{h} \mathcal{S}_{\epsilon} v-\mathcal{S}_{\epsilon} v\right)+\left(\mathcal{S}_{\epsilon} v-v\right)+\left(g-I_{h} P_{h} g\right)\right\|_{L^{2}(\Omega)} \\
& \quad+\left\|\left(I_{h} P_{h} \mathcal{S}_{\epsilon} v-\mathcal{S}_{\epsilon} v\right)+\left(\mathcal{S}_{\epsilon} v-v\right)+\left(g-I_{h} P_{h} g\right)\right\|_{L^{2}(\Omega)}^{2} . \\
& \leq\|v-g\|_{L^{2}(\Omega)}^{2}+2\|v-g\|_{L^{2}(\Omega)} \\
& \quad \times\left(\left\|I_{h} P_{h} \mathcal{S}_{\epsilon} v-\mathcal{S}_{\epsilon} v\right\|_{L^{2}(\Omega)}+\left\|\mathcal{S}_{\epsilon} v-v\right\|_{L^{2}(\Omega)}+\left\|g-I_{h} P_{h} g\right\|_{L^{2}(\Omega)}\right) \\
& \quad+C\left(\left\|I_{h} P_{h} \mathcal{S}_{\epsilon} v-\mathcal{S}_{\epsilon} v\right\|_{L^{2}(\Omega)}^{2}+\left\|\mathcal{S}_{\epsilon} v-v\right\|_{L^{2}(\Omega)}^{2}+\left\|g-I_{h} P_{h} g\right\|_{L^{2}(\Omega)}^{2}\right) .
\end{aligned}
$$

From (1.37) we can bound

$$
\left\|\mathcal{S}_{\epsilon} v-v\right\|_{L^{2}(\Omega)} \leq C \omega(v, \epsilon)_{L^{2}(\Omega)}
$$

and from (1.24) we know that

$$
\left\|I_{h} P_{h} g-g\right\|_{L^{2}(\Omega)} \leq C \omega(g, h)_{L^{2}(\Omega)} .
$$


We also have from (1.24) and (1.35)

$$
\left\|I_{h} P_{h} \mathcal{S}_{\epsilon} v-\mathcal{S}_{\epsilon} v\right\|_{L^{2}(\Omega)} \leq C \omega\left(\mathcal{S}_{\epsilon} v, h\right)_{L^{2}(\Omega)} \leq C \omega(v, h)_{L^{2}(\Omega)} .
$$

Thus,

$$
\begin{aligned}
\left\|P_{h} \mathcal{S}_{\epsilon} v-P_{h} g\right\|_{L^{2}\left(\Omega^{h}\right)}^{2} & \leq\|v-g\|_{L^{2}(\Omega)}^{2}+C\|v-g\|_{L^{2}(\Omega)} \\
& \times\left(\omega(v, h)_{L^{2}(\Omega)}+\omega(v, \epsilon)_{L^{2}(\Omega)}+\omega(g, h)_{L^{2}(\Omega)}\right) \\
& +C\left(\omega(v, h)_{L^{2}(\Omega)}^{2}+\omega(v, \epsilon)_{L^{2}(\Omega)}^{2}+\omega(g, h)_{L^{2}(\Omega)}^{2}\right) .
\end{aligned}
$$

Using this inequality as well as (2.11) in (2.10) yields (2.8).

Now let $v^{h}$ be any discrete function. Then

$$
E\left(\operatorname{Int} S_{L} v^{h}\right)=\left|\operatorname{Int} S_{L} v^{h}\right|_{\mathrm{BV}(\Omega)}+\frac{1}{2 \lambda}\left\|\operatorname{Int} S_{L} v^{h}-g\right\|_{L^{2}(\Omega)}^{2} .
$$

By (2.6), the first term on the right is bounded by

$$
J_{*}\left(v^{h}\right)+\frac{C}{L} J_{*}\left(v^{h}\right) .
$$

Now

$$
\begin{aligned}
& \| \text { Int } S_{L} v^{h}-g \|_{L^{2}(\Omega)}^{2} \\
& =\|\left(\operatorname{Int} S_{L} v^{h}-I_{h} S_{L} v^{h}\right)+\left(I_{h} S_{L} v^{h}-I_{h} v^{h}\right) \\
& \quad+\left(I_{h} v^{h}-I_{h} P_{h} g\right)+\left(I_{h} P_{h} g-g\right) \|_{L^{2}(\Omega)}^{2} \\
& \leq\left\|I_{h} v^{h}-I_{h} P_{h} g\right\|_{L^{2}(\Omega)}^{2}+2\left\|I_{h} v^{h}-I_{h} P_{h} g\right\|_{L^{2}(\Omega)} \\
& \quad \times\left\|\left(\operatorname{Int} S_{L} v^{h}-I_{h} S_{L} v^{h}\right)+\left(I_{h} S_{L} v^{h}-I_{h} v^{h}\right)+\left(I_{h} P_{h} g-g\right)\right\|_{L^{2}(\Omega)} \\
& \quad+\left\|\left(\operatorname{Int} S_{L} v^{h}-I_{h} S_{L} v^{h}\right)+\left(I_{h} S_{L} v^{h}-I_{h} v^{h}\right)+\left(I_{h} P_{h} g-g\right)\right\|_{L^{2}(\Omega)}^{2} \\
& \leq\left\|I_{h} v^{h}-I_{h} P_{h} g\right\|_{L^{2}(\Omega)}^{2}+2\left\|I_{h} v^{h}-I_{h} P_{h} g\right\|_{L^{2}(\Omega)} \\
& \quad \times\left(\left\|\operatorname{Int} S_{L} v^{h}-I_{h} S_{L} v^{h}\right\|_{L^{2}(\Omega)}+\left\|I_{h} S_{L} v^{h}-I_{h} v^{h}\right\|_{L^{2}(\Omega)}+\left\|I_{h} P_{h} g-g\right\|_{L^{2}(\Omega)}\right) \\
& \quad+C\left(\left\|\operatorname{Int} S_{L} v^{h}-I_{h} S_{L} v^{h}\right\|_{L^{2}(\Omega)}^{2}+\left\|I_{h} S_{L} v^{h}-I_{h} v^{h}\right\|_{L^{2}(\Omega)}^{2}+\left\|I_{h} P_{h} g-g\right\|_{L^{2}(\Omega)}^{2}\right)
\end{aligned}
$$

Since, for all discrete $v^{h},\left\|I_{h} v^{h}\right\|_{L^{2}(\Omega)}=\left\|v^{h}\right\|_{L^{2}\left(\Omega^{h}\right)}$, the quantity above is bounded by

$$
\begin{aligned}
& \left\|v^{h}-P_{h} g\right\|_{L^{2}\left(\Omega^{h}\right)}^{2}+2\left\|v^{h}-P_{h} g\right\|_{L^{2}\left(\Omega^{h}\right)} \\
& \quad \times\left(\left\|\operatorname{Int} S_{L} v^{h}-I_{h} S_{L} v^{h}\right\|_{L^{2}(\Omega)}+\left\|S_{L} v^{h}-v^{h}\right\|_{L^{2}\left(\Omega^{h}\right)}+\left\|I_{h} P_{h} g-g\right\|_{L^{2}(\Omega)}\right) \\
& \quad+C\left(\left\|\operatorname{Int} S_{L} v^{h}-I_{h} S_{L} v^{h}\right\|_{L^{2}(\Omega)}^{2}+\left\|S_{L} v^{h}-v^{h}\right\|_{L^{2}\left(\Omega^{h}\right)}^{2}+\left\|I_{h} P_{h} g-g\right\|_{L^{2}(\Omega)}^{2}\right)
\end{aligned}
$$

From (1.36) we have

$$
\left\|S_{L} v^{h}-v^{h}\right\|_{L^{2}\left(\Omega^{h}\right)} \leq C \omega\left(v^{h}, L\right)_{L^{2}\left(\Omega^{h}\right)} .
$$

By (1.28) and (1.34) we have

$$
\begin{aligned}
\left\|\operatorname{Int} S_{L} v^{h}-I_{h} S_{L} v^{h}\right\|_{L^{2}(\Omega)} & \leq C \omega\left(S_{L} v^{h}, 1\right)_{L^{2}\left(\Omega^{h}\right)} \\
& \leq C \omega\left(v^{h}, 1\right)_{L^{2}\left(\Omega^{h}\right)} \leq C \omega\left(v^{h}, L\right)_{L^{2}\left(\Omega^{h}\right)} .
\end{aligned}
$$


Combining these inequalities, we have

$$
\begin{aligned}
\left\|\operatorname{Int} S_{L} v^{h}-g\right\|_{L^{2}(\Omega)}^{2} \leq & \left\|v^{h}-P_{h} g\right\|_{L^{2}\left(\Omega^{h}\right)}^{2} \\
& +C\left\|v^{h}-P_{h} g\right\|_{L^{2}\left(\Omega^{h}\right)}\left(\omega\left(v^{h}, L\right)_{L^{2}\left(\Omega^{h}\right)}+\omega(g, h)_{L^{2}(\Omega)}\right) \\
& +C\left(\omega\left(v^{h}, L\right)_{L^{2}(\Omega)}^{2}+\omega(g, h)_{L^{2}(\Omega)}^{2}\right) .
\end{aligned}
$$

Combining this inequality with (2.12) and (2.13) yields (2.9).

3. Properties of the continuous and discrete minimizers. We need to discuss some properties of minimizers of the discrete and continuous functionals. We begin by comparing functionals on $\Omega$ and $\Omega^{h}$ and the corresponding functionals on $2 \Omega$ and $2 \Omega^{h}$. We remind the reader of the notations used in Lemma 1.2.

LEMma 3.1 (Extending minimizers). If $u^{h}$ is the minimizer of the functional

$$
E_{h}^{\Omega^{h}}\left(v^{h}\right)=E_{h}\left(v^{h}\right)=\frac{1}{2 \lambda}\left\|v^{h}-g^{h}\right\|_{L^{2}\left(\Omega^{h}\right)}^{2}+J_{*}^{\Omega^{h}}\left(v^{h}\right),
$$

then $\operatorname{Ext}_{h} u^{h}$ is the minimizer over all discrete functions $v^{h}$ defined on $2 \Omega^{h}$ of the functional

$$
E_{h}^{2 \Omega^{h}}\left(v^{h}\right)=\frac{1}{2 \lambda}\left\|v^{h}-\operatorname{Ext}_{h} g^{h}\right\|_{L^{2}\left(2 \Omega^{h}\right)}^{2}+J_{*}^{2 \Omega^{h}}\left(v^{h}\right)
$$

with periodic boundary conditions.

Similarly, if $u$ is the minimizer of

$$
E^{\Omega}(v)=E(v)=\frac{1}{2 \lambda}\|v-g\|_{L^{2}(\Omega)}^{2}+|v|_{\mathrm{BV}(\Omega)}
$$

then Ext $u$ is the minimizer of

$$
E^{2 \Omega}(v)=\frac{1}{2 \lambda}\|v-\operatorname{Ext} g\|_{L^{2}(2 \Omega)}^{2}+|v|_{\mathrm{BV}(2 \Omega)},
$$

again with periodic boundary conditions.

Furthermore, if $u$ and $w$ are minimizers of (3.3) with data $g$ and $h$, respectively, then

$$
\|u-w\|_{L^{2}(\Omega)} \leq\|g-h\|_{L^{2}(\Omega)} ;
$$

similarly for the discrete and continuous minimizers of (3.1)-(3.4). Thus, for the two periodic problems (3.2) and (3.4) we have

$$
\left\|\operatorname{Ext}_{h} u^{h}-T_{\ell} \operatorname{Ext}_{h} u^{h}\right\|_{L^{2}\left(2 \Omega^{h}\right)} \leq\left\|\operatorname{Ext}_{h} g^{h}-T_{\ell} \operatorname{Ext}_{h} g^{h}\right\|_{L^{2}\left(2 \Omega^{h}\right)}
$$

and

$$
\left\|\operatorname{Ext} u-\mathcal{T}_{\tau} \operatorname{Ext} u\right\|_{L^{2}(2 \Omega)} \leq\left\|\operatorname{Ext} g-\mathcal{T}_{\tau} \operatorname{Ext} g\right\|_{L^{2}(2 \Omega)} .
$$

Proof. To avoid confusion, we use letters with bars to indicate functions defined on $2 \Omega^{h}$, for example $\bar{v}^{h}$, in the proof. Moreover we remind the reader for any discrete function $f^{h}$ defined on $\Omega^{h}$, the extended function $\operatorname{Ext}_{h} f^{h}$ is also defined on $2 \Omega^{h}$. 
Note that the discrete set $2 \Omega^{h}$ is the union of four quadrants,

$$
2 \Omega^{h}=\bigcup_{i=1}^{4} \Omega_{i}^{h}
$$

where $\Omega_{1}^{h}=\Omega^{h}, \Omega_{2}^{h}=\Omega^{h}+(N, 0), \Omega_{3}^{h}=\Omega^{h}+(0, N), \Omega_{4}^{h}=\Omega^{h}+(N, N)$.

For discrete functions $f^{h}$ defined on a quadrant $A \in\left\{\Omega_{1}^{h}, \Omega_{2}^{h}, \Omega_{3}^{h}, \Omega_{4}^{h}\right\}$, we define $J_{*}^{A}$ in the same way as defining $J_{*}^{\Omega^{h}}$ with Neumann boundary condition imposed. We also define $\operatorname{Ext}_{h} f^{h}$ the same way as extending functions defined on $\Omega^{h}$ to $\mathbb{Z}^{2}$.

For discrete functions $\bar{v}^{h}$ defined on $2 \Omega^{h}$ and any quadrant $A$, we define the discrete variation restricted on $A$ of $\bar{v}^{h}$ by

$$
\left.J_{\oplus \ominus}^{2 \Omega^{h}}\left(\bar{v}^{h}\right)\right|_{A}=\sum_{i \in A} \sqrt{\left(\frac{\bar{v}_{i \oplus(1,0)}^{h}-\bar{v}_{i}^{h}}{h}\right)^{2}+\left(\frac{\bar{v}_{i \ominus(0,1)}^{h}-\bar{v}_{i}^{h}}{h}\right)^{2}} h^{2}
$$

with $\oplus, \ominus \in\{+,-\}$, and the symmetric discrete variation restricted on $A$ by

$$
\left.J_{*}^{2 \Omega^{h}}\left(\bar{v}^{h}\right)\right|_{A}=\frac{1}{4}\left(\left.J_{++}^{2 \Omega^{h}}\left(\bar{v}^{h}\right)\right|_{A}+\left.J_{+-}^{2 \Omega^{h}}\left(\bar{v}^{h}\right)\right|_{A}+\left.J_{-+}^{2 \Omega^{h}}\left(\bar{v}^{h}\right)\right|_{A}+\left.J_{--}^{2 \Omega^{h}}\left(\bar{v}^{h}\right)\right|_{A}\right) .
$$

For variations restricted on a quadrant, we use periodic boundary condition if the values of $\bar{v}_{i}^{h}$ with $i$ outside $2 \Omega^{h}$ are needed. In fact, $\left.J_{*}^{2 \Omega^{h}}\right|_{A}$ is only a fraction of $J_{*}^{2 \Omega^{h}}$ where the sum is taken over the subset $A$ of $2 \Omega^{h}$.

We point out that we have introduced two kinds of variations defined either on a quadrant $A$ or on $2 \Omega^{h}$. They use two different boundary conditions. $J_{*}^{A}$ uses Neumann boundary condition that sets variation terms "across" the boundary zero; $J_{*}^{2 \Omega^{h}}$ (or the restricted $\left.J_{*}^{2 \Omega^{h}}\right|_{A}$ ) uses periodic boundary conditions that assumes the values of $\bar{v}^{h}$ are periodically extended to $\mathbb{Z}^{2}$ with period $(2 N, 2 N)$.

Moreover, we define the corresponding functional restricted on $A$ by

$$
\left.E_{h}^{2 \Omega^{h}}\left(\bar{v}^{h}\right)\right|_{A}=\left.J_{*}^{2 \Omega^{h}}\left(\bar{v}^{h}\right)\right|_{A}+\frac{1}{2 \lambda}\left\|\bar{v}^{h}-\operatorname{Ext}_{h} g^{h}\right\|_{L^{2}(A)}^{2} .
$$

Similarly $\left.E_{h}^{2 \Omega^{h}}\right|_{A}$ is a fraction of $E_{h}^{2 \Omega^{h}}$ where the sum is taken over $A$. Thus it is trivial

$$
\left.\sum_{i=1}^{4} E_{h}^{2 \Omega^{h}}\left(\bar{v}^{h}\right)\right|_{\Omega_{i}^{h}}=E_{h}^{2 \Omega^{h}}\left(\bar{v}^{h}\right)
$$

At last for any $\bar{v}^{h}$ defined on $2 \Omega^{h}$, we use $\left.\bar{v}\right|_{A}$ to indicate a discrete function defined on $A$ that equals $\bar{v}^{h}$ restricted on $A$.

We show that

$$
J_{*}^{A}\left(\left.\bar{v}^{h}\right|_{A}\right) \leq\left. J_{*}^{2 \Omega^{h}}\left(\bar{v}^{h}\right)\right|_{A},
$$

and

$$
E_{h}^{A}\left(\left.\bar{v}^{h}\right|_{A}\right) \leq\left. E_{h}^{2 \Omega^{h}}\left(\bar{v}^{h}\right)\right|_{A},
$$

This is because, letting the boundary of $A$ to be the set

$$
\partial A:=\left\{\left(i_{1}, i_{2}\right) \mid i_{1} \text { or } \mathrm{i}_{2} \in\{0, \mathrm{~N}-1, \mathrm{~N}, 2 \mathrm{~N}-1\}\right\},
$$


$\left.J_{*}^{2 \Omega^{h}}\left(\bar{v}^{h}\right)\right|_{A}$ and $J_{*}^{A}\left(\left.\bar{v}^{h}\right|_{A}\right)$ both include the sum of variation terms at inner points of $A$, i.e., $A \backslash \partial A$; for the sum taken over $\partial A$ where variations include differences of $\bar{v}^{h}$ at points both inside and outside $A,\left.J_{*}^{2 \Omega^{h}}\left(\bar{v}^{h}\right)\right|_{A}$ assumes $\bar{v}^{h}$ outside $A$ satisfies periodic boundary condition, while $J_{*}^{A}\left(\left.\bar{v}^{h}\right|_{A}\right)$ simply assumes such differences equal zero. Hence we get (3.8). (3.9) is a straightforward deduction of (3.8).

Note that for the extended function $\operatorname{Ext}_{h} f^{h}$ of $f^{h}$ that is defined on a quadrant, these equations hold due to the reflection in extending.

$$
\begin{array}{ll}
J_{*}^{\Omega_{i}^{h}}\left(\left.\operatorname{Ext}_{h} f^{h}\right|_{\Omega_{i}^{h}}\right)=\left.J_{*}^{2 \Omega^{h}}\left(\operatorname{Ext}_{h} f^{h}\right)\right|_{\Omega_{i}^{h}}, \quad 1 \leq i \leq 4 \\
E_{h}^{\Omega_{i}^{h}}\left(\left.\operatorname{Ext}_{h} f^{h}\right|_{\Omega_{i}^{h}}\right)=\left.E_{h}^{2 \Omega^{h}}\left(\operatorname{Ext}_{h} f^{h}\right)\right|_{\Omega_{i}^{h} .} \quad 1 \leq i \leq 4
\end{array}
$$

Also by the symmetric nature of $J_{*}^{A}$ and the definition of $\mathrm{Ext}_{h}$, it is trivial

$$
J_{*}^{\Omega_{j}^{h}}\left(\left.\operatorname{Ext}_{h} f^{h}\right|_{\Omega_{j}^{h}}\right)=J_{*}^{\Omega_{i}^{h}}\left(f^{h}\right) \quad 1 \leq j \leq 4, j \neq i
$$

and

$$
\left\|\operatorname{Ext}_{h} f^{h}-\operatorname{Ext}_{h} g^{h}\right\|_{L^{2}\left(\Omega_{j}^{h}\right)}=\left\|\operatorname{Ext}_{h} f^{h}-\operatorname{Ext}_{h} g^{h}\right\|_{L^{2}\left(\Omega_{i}^{h}\right)} . \quad 1 \leq j \leq 4, j \neq i
$$

Therefore, on any quadrant $\Omega_{i}^{h}, 1 \leq i \leq 4$, the minimization problem

$$
\arg \min _{w^{h} \in L^{2}\left(\Omega^{h}\right)} E_{h}^{\Omega_{i}^{h}}\left(w^{h}\right)=\arg \min _{w^{h} \in L^{2}\left(\Omega^{h}\right)} J_{*}^{\Omega_{i}^{h}}\left(w^{h}\right)+\frac{1}{2 \lambda}\left\|w^{h}-\operatorname{Ext}_{h} g^{h}\right\|_{L^{2}\left(\Omega_{i}^{h}\right)}^{2}
$$

has a unique solution $\left.\operatorname{Ext}_{h} u^{h}\right|_{\Omega_{i}^{h}}$, because for any discrete function $w^{h}$ defined on $\Omega_{i}^{h}$

$$
\begin{aligned}
& J_{*}^{\Omega_{i}^{h}}\left(w^{h}\right)+\frac{1}{2 \lambda}\left\|w^{h}-\operatorname{Ext}_{h} g^{h}\right\|_{L^{2}\left(\Omega_{i}^{h}\right)}^{2} \\
= & J_{*}^{\Omega^{h}}\left(\left.\left(\operatorname{Ext}_{h} w^{h}\right)\right|_{\Omega^{h}}\right)+\frac{1}{2 \lambda}\left\|\operatorname{Ext}_{h} w^{h}-g^{h}\right\|_{L^{2}\left(\Omega^{h}\right)}^{2} \quad \text { by }(3.12) \text { and }(3.13) \\
\geq & J_{*}^{\Omega^{h}}\left(u^{h}\right)+\frac{1}{2 \lambda}\left\|u^{h}-g^{h}\right\|_{L^{2}\left(\Omega^{h}\right)}^{2} \\
= & J_{*}^{\Omega_{i}^{h}}\left(\left.\left(\operatorname{Ext}_{h} u^{h}\right)\right|_{\Omega_{i}^{h}}\right)+\frac{1}{2 \lambda}\left\|\operatorname{Ext}_{h} u^{h}-\operatorname{Ext}_{h} g^{h}\right\|_{L^{2}\left(\Omega_{i}^{h}\right)}^{2} . \quad \text { by (3.12) and }(3 .
\end{aligned}
$$

Thus for any discrete function $\bar{v}^{h}$ defined on $2 \Omega^{h}$,

$$
E_{h}^{\Omega_{i}^{h}}\left(\left.\operatorname{Ext}_{h} u^{h}\right|_{\Omega_{i}^{h}}\right) \leq E_{h}^{\Omega_{i}^{h}}\left(\left.\bar{v}^{h}\right|_{\Omega_{i}^{h}}\right) . \quad 1 \leq i \leq 4
$$

Adding this inequality for $i=1,2,3,4$ and noting by (3.11)

$$
\sum_{i=1}^{4} E_{h}^{\Omega_{i}^{h}}\left(\left.\operatorname{Ext}_{h} u^{h}\right|_{\Omega_{i}^{h}}\right)=E_{h}^{2 \Omega^{h}}\left(\operatorname{Ext}_{h} u^{h}\right),
$$

we have

$$
E_{h}^{2 \Omega^{h}}\left(\operatorname{Ext}_{h} u^{h}\right) \leq \sum_{i=1}^{4} E_{h}^{\Omega_{i}^{h}}\left(\left.\bar{v}^{h}\right|_{\Omega_{i}^{h}}\right)
$$


For each term on the right hand, by (3.9)

$$
E_{h}^{\Omega_{i}^{h}}\left(\left.\bar{v}^{h}\right|_{\Omega_{i}^{h}}\right) \leq\left. E_{h}^{2 \Omega^{h}}\left(\bar{v}^{h}\right)\right|_{\Omega_{i}^{h}} .
$$

Then adding them all, we have for any discrete function $\bar{v}^{h}$ defined on $2 \Omega^{h}$,

$$
E_{h}^{2 \Omega^{h}}\left(\operatorname{Ext}_{h} u^{h}\right) \leq\left.\sum_{i=1}^{4} E_{h}^{2 \Omega^{h}}\left(\bar{v}^{h}\right)\right|_{\Omega_{i}^{h}}=E_{h}^{2 \Omega^{h}}\left(\bar{v}^{h}\right) \quad \text { by }(3.7)
$$

i.e., $\operatorname{Ext}_{h} u^{h}$ is the minimizer of the functional (3.2).

The second part is the continuous analogue of the discrete case. And the last two inequalities are standard. $\square$

The next lemma is classical.

Lemma 3.2 (Smoothness bounds). Assume $u$ is the minimizer of the functional

$$
E(v)=\frac{1}{2 \lambda}\|v-g\|_{L^{2}(\Omega)}^{2}+|v|_{\mathrm{BV}(\Omega)}
$$

and $u^{h}$ is the minimizer of the discrete functional

$$
E_{h}\left(v^{h}\right)=\frac{1}{2 \lambda}\left\|v^{h}-P_{h} g\right\|_{L^{2}\left(\Omega^{h}\right)}^{2}+J_{h}\left(v^{h}\right) .
$$

Then

$$
\|u-g\|_{L^{2}(\Omega)} \leq\|g\|_{L^{2}(\Omega)}
$$

and

$$
\left\|u^{h}-P_{h} g\right\|_{L^{2}\left(\Omega^{h}\right)} \leq\left\|P_{h} g\right\|_{L^{2}\left(\Omega^{h}\right)} \leq\|g\|_{L^{2}(\Omega)} .
$$

Additionally, we have

$$
|u|_{\mathrm{BV}(\Omega)} \leq \frac{1}{2 \lambda}\|g\|_{L^{2}(\Omega)}^{2}
$$

and

$$
J_{h}\left(u^{h}\right) \leq \frac{1}{2 \lambda}\left\|P_{h} g\right\|_{L^{2}\left(\Omega^{h}\right)}^{2} \leq \frac{1}{2 \lambda}\|g\|_{L^{2}(\Omega)}^{2} .
$$

We also have

$$
\omega(u, \epsilon)_{L^{2}(\Omega)} \leq C \omega(g, \epsilon)_{L^{2}(\Omega)}
$$

and if $J_{h}=J_{*}$ then

$$
\omega\left(u^{h}, L\right)_{L^{2}\left(\Omega^{h}\right)} \leq C \omega\left(P_{h} g, L\right)_{L^{2}\left(\Omega^{h}\right)} \leq C \omega(g, L h)_{L^{2}(\Omega)} .
$$

Proof. The results are classical, but some of the proofs are short, so we give them here.

We know that $E(u) \leq E(0)$, so

$$
\frac{1}{2 \lambda}\|u-g\|_{L^{2}(\Omega)}^{2} \leq \frac{1}{2 \lambda}\|u-g\|_{L^{2}(\Omega)}^{2}+|u|_{\mathrm{BV}(\Omega)} \leq \frac{1}{2 \lambda}\|g\|_{L^{2}(\Omega)}^{2} .
$$


One proves (3.15) in the same way.

Inequalities (3.16) and (3.17) follow from $E(u) \leq E(0)$ and $E_{h}\left(u^{h}\right) \leq E_{h}(0)$, respectively.

For inequality (3.18), by definition

$$
\begin{aligned}
\omega(u, \epsilon)_{L^{2}(\Omega)} & \leq \sup _{\tau \in \mathbb{R}^{2},|\tau|<\epsilon}\left(\int_{x, x+\tau \in \Omega}|u(x+\tau)-u(x)|^{2} d x\right)^{\frac{1}{2}} \\
& \leq \sup _{\tau \in \mathbb{R}^{2},|\tau|<\epsilon}\left(\int_{x \in 2 \Omega}|\operatorname{Ext} u(x+\tau)-\operatorname{Ext} u(x)|^{2} d x\right)^{\frac{1}{2}}
\end{aligned}
$$

By (3.6)

$$
\begin{aligned}
\omega(u, \epsilon)_{L^{2}(\Omega)} & \leq \sup _{\tau \in \mathbb{R}^{2},|\tau|<\epsilon}\left(\int_{x \in 2 \Omega}|\operatorname{Ext} g(x+\tau)-\operatorname{Ext} g(x)|^{2} d x\right)^{\frac{1}{2}} \\
& \leq C \omega(g, \epsilon)_{L^{2}(2 \Omega)} .
\end{aligned}
$$

The last line follows from (1.4) of Lemma 1.1.

It is similar to prove the left part of (3.19) by using the definition of $\omega\left(u^{h}, L\right)_{L^{2}\left(\Omega^{h}\right)}$ and applying (3.5) and (1.5). The right part of (3.19) is just (1.21).

4. Proof of the main theorems. We now bound the difference between discrete and continuous functionals at their respective minimizers.

THEOREM 4.1 (Functional difference). Assume $u$ is the minimizer of the functional

$$
E(v)=\frac{1}{2 \lambda}\|v-g\|_{L^{2}(\Omega)}^{2}+|v|_{\mathrm{BV}(\Omega)}
$$

for $g \in \operatorname{Lip}\left(\alpha, L^{2}(\Omega)\right)$ and $u^{h}$ is the minimizer of the discrete functional

$$
E_{h}\left(v^{h}\right)=\frac{1}{2 \lambda}\left\|v^{h}-P_{h} g\right\|_{L^{2}\left(\Omega^{h}\right)}^{2}+J_{*}\left(v^{h}\right) .
$$

Then if $\epsilon=h^{1 /(\alpha+1)}$ we have

$$
E_{h}\left(P_{h} \mathcal{S}_{\epsilon} u\right) \leq E(u)+\frac{C}{\lambda}\|g\|_{\operatorname{Lip}\left(\alpha, L^{2}(\Omega)\right)}^{2} h^{\alpha /(\alpha+1)} .
$$

and if $L$ is set to the integer part of $h^{-\alpha /(\alpha+1)}$ then

$$
E\left(\operatorname{Int} S_{L} u^{h}\right) \leq E_{h}\left(u^{h}\right)+\frac{C}{\lambda}\|g\|_{\operatorname{Lip}\left(\alpha, L^{2}(\Omega)\right)}^{2} h^{\alpha /(\alpha+1)} .
$$

Finally,

$$
\left|E(u)-E_{h}\left(u^{h}\right)\right| \leq \frac{C}{\lambda}\|g\|_{\operatorname{Lip}\left(\alpha, L^{2}(\Omega)\right)}^{2} h^{\alpha /(\alpha+1)}
$$

Proof. We mainly use Lemma 2.3 and Lemma 3.2. By (2.8) of Lemma 2.3

$$
\begin{aligned}
E_{h}\left(P_{h} \mathcal{S}_{\epsilon} u\right) & \leq E(u)+\frac{C h}{\epsilon}|u|_{\mathrm{BV}(\Omega)} \\
& +\frac{C}{\lambda}\|u-g\|_{L^{2}(\Omega)}\left(\omega(u, h)_{L^{2}(\Omega)}+\omega(u, \epsilon)_{L^{2}(\Omega)}+\omega(g, h)_{L^{2}(\Omega)}\right) \\
& +\frac{C}{\lambda}\left(\omega(u, h)_{L^{2}(\Omega)}^{2}+\omega(u, \epsilon)_{L^{2}(\Omega)}^{2}+\omega(g, h)_{L^{2}(\Omega)}^{2}\right)
\end{aligned}
$$


We then apply (3.14), (3.16), and (3.18) to obtain

$$
\begin{aligned}
E_{h}\left(P_{h} \mathcal{S}_{\epsilon} u\right) & \leq E(u)+\frac{C h}{\epsilon \lambda}\|g\|_{L^{2}(\Omega)}^{2} \\
& +\frac{C}{\lambda}\|g\|_{L^{2}(\Omega)}\left(\omega(g, \epsilon)_{L^{2}(\Omega)}+\omega(g, h)_{L^{2}(\Omega)}\right) \\
& +\frac{C}{\lambda}\left(\omega(g, h)_{L^{2}(\Omega)}^{2}+\omega(g, \epsilon)_{L^{2}(\Omega)}^{2}\right) .
\end{aligned}
$$

Since $g \in \operatorname{Lip}\left(\alpha, L^{2}(\Omega)\right)$,

$$
\omega(g, t)_{L^{2}(\Omega)} \leq|g|_{\operatorname{Lip}\left(\alpha, L^{2}(\Omega)\right)} t^{\alpha}, \quad t>0 .
$$

Thus

$$
\begin{aligned}
E_{h}\left(P_{h} \mathcal{S}_{\epsilon} u\right) & \leq E(u)+\frac{C h}{\epsilon \lambda}\|g\|_{L^{2}(\Omega)}^{2} \\
& +\frac{C}{\lambda}\|g\|_{L^{2}(\Omega)}|g|_{\operatorname{Lip}\left(\alpha, L^{2}(\Omega)\right)}\left(\epsilon^{\alpha}+h^{\alpha}\right) \\
& +\frac{C}{\lambda}|g|_{\operatorname{Lip}\left(\alpha, L^{2}(\Omega)\right)}^{2}\left(\epsilon^{2 \alpha}+h^{2 \alpha}\right) \\
& \leq E(u)+\frac{C}{\lambda}\|g\|_{\operatorname{Lip}\left(\alpha, L^{2}(\Omega)\right)}^{2}\left(\frac{h}{\epsilon}+\epsilon^{\alpha}+h^{\alpha}+\epsilon^{2 \alpha}+h^{2 \alpha}\right)
\end{aligned}
$$

We know at a minimum $1>\epsilon>h$, so setting the largest error terms $h / \epsilon$ and $\epsilon^{\alpha}$ equal, i.e., setting $\epsilon=h^{1 /(\alpha+1)}$, we have

$$
E_{h}\left(P_{h} \mathcal{S}_{\epsilon} u\right) \leq E(u)+\frac{C}{\lambda}\|g\|_{\operatorname{Lip}\left(\alpha, L^{2}(\Omega)\right)}^{2}\left(h^{\alpha /(\alpha+1)}+h^{\alpha}+h^{2 \alpha}+h^{2 \alpha /(\alpha+1)}\right) .
$$

Thus we obtain (4.1).

We point out that (4.1) holds for any discrete variation $J_{h}$ defined in (1.9). More generally it holds for any discrete variation satisfying Lemma 2.2 .

Similarly, if one begins with (2.9) and applies (3.15), (3.17), and (3.19), one finds on setting $L$ to the integer part of $h^{-\alpha /(\alpha+1)}$ that

$$
E\left(\operatorname{Int} S_{L} u^{h}\right) \leq E_{h}\left(u^{h}\right)+\frac{C}{\lambda}\|g\|_{\operatorname{Lip}\left(\alpha, L^{2}(\Omega)\right)}^{2} h^{\alpha /(\alpha+1)},
$$

which is (4.2).

Because $u$ and $u^{h}$ are minimizers of their respective functionals, we have

$$
E_{h}\left(u^{h}\right) \leq E_{h}\left(P_{h} \mathcal{S}_{\epsilon} u\right) \leq E(u)+\frac{C}{\lambda}\|g\|_{\operatorname{Lip}\left(\alpha, L^{2}(\Omega)\right)}^{2} h^{\alpha /(\alpha+1)}
$$

and

$$
E(u) \leq E\left(\operatorname{Int} S_{L} u^{h}\right) \leq E_{h}\left(u^{h}\right)+\frac{C}{\lambda}\|g\|_{\operatorname{Lip}\left(\alpha, L^{2}(\Omega)\right)}^{2} h^{\alpha /(\alpha+1)} .
$$

Then (4.3) is proved.

To show the error bound for minimizers, we need the following result, which can be proved easily using classical arguments.

Lemma 4.1. If $u$ is the minimizer of the functional $E(v)$ defined in Theorem 4.1, then for any $v \in \mathrm{BV}(\Omega)$

$$
\|v-u\|_{L^{2}(\Omega)}^{2} \leq 2 \lambda(E(v)-E(u)) .
$$


Moreover, if $u^{h}$ is the minimizer of $E_{h}\left(v^{h}\right)$, then for any $v^{h}$ defined on $\Omega^{h}$

$$
\left\|v^{h}-u^{h}\right\|_{L^{2}\left(\Omega^{h}\right)}^{2} \leq 2 \lambda\left(E_{h}\left(v^{h}\right)-E_{h}\left(u^{h}\right)\right) .
$$

Theorem 4.2 (Minimizer difference). Assume $u$ is the minimizer of the functional

$$
E(v)=\frac{1}{2 \lambda}\|v-g\|_{L^{2}(\Omega)}^{2}+|v|_{\mathrm{BV}(\Omega)}
$$

for $g \in \operatorname{Lip}\left(\alpha, L^{2}(\Omega)\right)$ and $u^{h}$ is the minimizer of the discrete functional

$$
E_{h}\left(v^{h}\right)=\frac{1}{2 \lambda}\left\|v^{h}-P_{h} g\right\|_{L^{2}\left(\Omega^{h}\right)}^{2}+J_{*}\left(v^{h}\right) .
$$

Then

$$
\left\|I_{h} u^{h}-u\right\|_{L^{2}(\Omega)}^{2} \leq C\|g\|_{\operatorname{Lip}\left(\alpha, L^{2}(\Omega)\right)}^{2} h^{\alpha /(\alpha+1)} .
$$

Proof. We apply (4.7) with $v^{h}=P_{h} \mathcal{S}_{\epsilon} u$ and $\epsilon=h^{1 /(\alpha+1)}$ :

$$
\begin{array}{rl}
\left\|P_{h} \mathcal{S}_{\epsilon} u-u^{h}\right\|_{L^{2}\left(\Omega^{h}\right) \leq}^{2} & 2 \lambda\left(E_{h}\left(P_{h} \mathcal{S}_{\epsilon} u^{h}\right)-E_{h}\left(u^{h}\right)\right) \\
\leq & 2 \lambda\left[\left(E(u)+\frac{C}{\lambda}\|g\|_{\operatorname{Lip}\left(\alpha, L^{2}(\Omega)\right)}^{2} h^{\alpha /(\alpha+1)}\right)\right. \\
& \left.+\left(-E(u)+\frac{C}{\lambda}\|g\|_{\operatorname{Lip}\left(\alpha, L^{2}(\Omega)\right)}^{2} h^{\alpha /(\alpha+1)}\right)\right] .
\end{array}
$$

The first substitution is by (4.1); the second is by (4.5). Thus we have

$$
\left\|P_{h} \mathcal{S}_{\epsilon} u-u^{h}\right\|_{L^{2}\left(\Omega^{h}\right)}^{2} \leq C\|g\|_{\operatorname{Lip}\left(\alpha, L^{2}(\Omega)\right)}^{2} h^{\alpha /(\alpha+1)} .
$$

Then

$$
\begin{aligned}
\left\|I_{h} u^{h}-u\right\|_{L^{2}(\Omega)}^{2} & =\left\|I_{h} u^{h}-I_{h} P_{h} \mathcal{S}_{\epsilon} u+I_{h} P_{h} \mathcal{S}_{\epsilon} u-\mathcal{S}_{\epsilon} u+\mathcal{S}_{\epsilon} u-u\right\|_{L^{2}(\Omega)}^{2} \\
& \leq 3\left(\left\|I_{h} u^{h}-I_{h} P_{h} \mathcal{S}_{\epsilon} u\right\|_{L^{2}(\Omega)}^{2}+\left\|I_{h} P_{h} \mathcal{S}_{\epsilon} u-\mathcal{S}_{\epsilon} u\right\|_{L^{2}(\Omega)}^{2}\right. \\
& \left.+\left\|\mathcal{S}_{\epsilon} u-u\right\|_{L^{2}(\Omega)}^{2}\right)
\end{aligned}
$$

We note by (1.22) and (4.8)

$$
\left\|I_{h} u^{h}-I_{h} P_{h} \mathcal{S}_{\epsilon} u\right\|_{L^{2}(\Omega)}^{2}=\left\|P_{h} \mathcal{S}_{\epsilon} u-u^{h}\right\|_{L^{2}\left(\Omega^{h}\right)}^{2} \leq C\|g\|_{\operatorname{Lip}\left(\alpha, L^{2}(\Omega)\right)}^{2} h^{\alpha /(\alpha+1)} .
$$

To bound $\left\|I_{h} P_{h} \mathcal{S}_{\epsilon} u-\mathcal{S}_{\epsilon} u\right\|_{L^{2}(\Omega)}$, by (1.24), (1.35), and (3.18), we have

$$
\begin{aligned}
\left\|I_{h} P_{h} \mathcal{S}_{\epsilon} u-\mathcal{S}_{\epsilon} u\right\|_{L^{2}(\Omega)} & \leq C \omega\left(\mathcal{S}_{\epsilon} u, h\right)_{L^{2}(\Omega)} \\
& \leq C \omega(u, h)_{L^{2}(\Omega)} \leq C \omega(g, h)_{L^{2}(\Omega)} .
\end{aligned}
$$

Finally by (1.37) and (3.18)

$$
\left\|\mathcal{S}_{\epsilon} u-u\right\|_{L^{2}(\Omega)} \leq C \omega(u, \epsilon) \leq C \omega(g, \epsilon) .
$$


Thus combining (4.8), (4.9) and (4.10), we have

$$
\begin{aligned}
\left\|I_{h} u^{h}-u\right\|_{L^{2}(\Omega)}^{2} & \leq C\left(\|g\|_{\operatorname{Lip}\left(\alpha, L^{2}(\Omega)\right)}^{2} h^{\alpha /(\alpha+1)}+\omega(g, h)_{L^{2}(\Omega)}^{2}+\omega(g, \epsilon)_{L^{2}(\Omega)}^{2}\right) \\
& \leq C\left(\|g\|_{\operatorname{Lip}\left(\alpha, L^{2}(\Omega)\right)}^{2} h^{\alpha /(\alpha+1)}+\|g\|_{L^{2}(\Omega)}^{2} h^{2 \alpha}+\|g\|_{L^{2}(\Omega)}^{2} h^{2 \alpha /(\alpha+1)}\right)
\end{aligned}
$$

Because the first term dominates the others, we have

$$
\left\|I_{h} u^{h}-u\right\|_{L^{2}(\Omega)}^{2} \leq C\|g\|_{\operatorname{Lip}\left(\alpha, L^{2}(\Omega)\right)}^{2} h^{\alpha /(\alpha+1)} .
$$

$\square$

5. Error bound for the upwind scheme. In this section, we prove the error bound for the "upwind" scheme. The "upwind" discrete gradient operator $-\nabla_{h}$ is defined by

$$
\left(-\nabla_{h}\right) v_{i}^{h}=\frac{1}{h}\left(\begin{array}{l}
\operatorname{Ext}_{h} v_{i}^{h}-\operatorname{Ext}_{h} v_{i+(1,0)}^{h} \\
\operatorname{Ext}_{h} v_{i}^{h}-\operatorname{Ext}_{h} v_{i-(1,0)}^{h} \\
\operatorname{Ext}_{h} v_{i}^{h}-\operatorname{Ext}_{h} v_{i+(0,1)}^{h} \\
\operatorname{Ext}_{h} v_{i}^{h}-\operatorname{Ext}_{h} v_{i-(0,1)}^{h}
\end{array}\right)
$$

The "upwind" discrete variation is then defined by

$$
J_{U}\left(v^{h}\right)=\sum_{i \in \Omega^{h}}\left|\left(-\nabla_{h}\right) v_{i}^{h} \vee 0\right| h^{2}
$$

where 0 is the vector $(0,0,0,0)$, and $p \vee q$ and $p \wedge q$ are componentwise maximum and minimum, respectively, of the vectors $p, q \in \mathbb{R}^{4}$.

In other words, we include a difference in the vector norm of the $i$ th term in (5.2) only if $v^{h}$ is increasing into $v_{i}^{h}$. Nothing changes in the following proofs (and one sees little change in the images themselves) if we change componentwise maximum ( $V$ ) to componentwise minimum $(\wedge)$ in (5.2). In their paper, Osher and Sethian [12] were solving Hamilton-Jacobi equations where this substitution could not be made: their problem, unlike ours, has a true notion of "wind".

To prove the result for the "upwind" scheme, we need to adapt to $J_{U}$ the previous lemmas involving $J_{*}$.

First we shall prove the convexity of $J_{U}$.

Lemma 5.1. $J_{U}$ is convex

Proof. First note that for two vectors $p, q \in \mathbb{R}^{n}$, it is easy to verify

$$
0 \leq(p+q) \vee 0 \leq p \vee 0+q \vee 0,
$$

where inequality $p \leq q$ means $p_{i} \leq q_{i}$ for each index $i$. Thus,

$$
|(p+q) \vee 0| \leq|p \vee 0|+|q \vee 0| \text {. }
$$

We apply (5.3) to each term in (5.2) of $J_{U}\left(\lambda f^{h}+(1-\lambda) g^{h}\right)$, where $1>\lambda>0$ and $f^{h}$ and $g^{h}$ are discrete functions, to find that

$$
\begin{aligned}
J_{U}\left(\lambda f^{h}+(1-\lambda) g^{h}\right) & =\sum_{i}\left|\left(-\nabla_{h}\right)\left(\lambda f^{h}+(1-\lambda) g^{h}\right)_{i} \vee 0\right| h^{2} \\
& \leq \sum_{i}\left\{\left|\lambda\left(-\nabla_{h}\right) f_{i}^{h} \vee 0\right|+\left|(1-\lambda)\left(-\nabla_{h}\right) g_{i}^{h} \vee 0\right|\right\} h^{2} \\
& =\lambda J_{U}\left(f^{h}\right)+(1-\lambda) J_{U}\left(g^{h}\right) .
\end{aligned}
$$


In the following we use the notation $\nabla_{x}^{+}$and $\nabla_{y}^{+}$defined in (2.3). We define similarly

$$
\nabla_{x}^{-} v_{i}^{h}=\frac{\operatorname{Ext}_{h} v_{i}^{h}-\operatorname{Ext}_{h} v_{i-(1,0)}^{h}}{h}, \quad \nabla_{y}^{-} v_{i}^{h}=\frac{\operatorname{Ext}_{h} v_{i}^{h}-\operatorname{Ext}_{h} v_{i-(0,1)}^{h}}{h} .
$$

Note that the divided differences are applied to the extended discrete function and that the difference is zero if $i \in \Omega^{h}$ and the other index is outside $\Omega^{h}$.

Using these operators, we can write

$$
J_{U}\left(v^{h}\right)=\sum_{i \in \Omega^{h}}\left|\left(\begin{array}{r}
-\nabla_{x}^{+} v_{i}^{h} \vee 0 \\
\nabla_{x}^{-} v_{i}^{h} \vee 0 \\
-\nabla_{y}^{+} v_{i}^{h} \vee 0 \\
\nabla_{y}^{-} v_{i}^{h} \vee 0
\end{array}\right)\right| h^{2} .
$$

The following lemma corresponds to Lemma 1.3.

Lemma 5.2. $J_{U}$ is equivalent to $|\cdot|_{W^{1,1}\left(\Omega^{h}\right)}$, where $|\cdot|_{W^{1,1}\left(\Omega^{h}\right)}$ is the discrete seminorm defined in (1.14). More precisely,

$$
\frac{1}{2}\left|v^{h}\right|_{W^{1,1}\left(\Omega^{h}\right)} \leq J_{U}\left(v^{h}\right) \leq\left|v^{h}\right|_{W^{1,1}\left(\Omega^{h}\right)}
$$

Proof. By (1.15)

$$
\begin{aligned}
& \frac{1}{2} \sum_{i}\left\{\left|-\nabla_{x}^{+} v_{i}^{h} \vee 0\right|+\left|\nabla_{x}^{-} v_{i}^{h} \vee 0\right|+\left|-\nabla_{y}^{+} v_{i}^{h} \vee 0\right|+\left|\nabla_{y}^{-} v_{i}^{h} \vee 0\right|\right\} h^{2} \\
& \leq \sum_{i} \sqrt{\left\{\left|-\nabla_{x}^{+} v_{i}^{h} \vee 0\right|^{2}+\left|\nabla_{x}^{-} v_{i}^{h} \vee 0\right|^{2}+\left|-\nabla_{y}^{+} v_{i}^{h} \vee 0\right|^{2}+\left|\nabla_{y}^{-} v_{i}^{h} \vee 0\right|^{2}\right\}} h^{2} \\
& \leq \sum_{i}\left\{\left|-\nabla_{x}^{+} v_{i}^{h} \vee 0\right|+\left|\nabla_{x}^{-} v_{i}^{h} \vee 0\right|+\left|-\nabla_{y}^{+} v_{i}^{h} \vee 0\right|+\left|\nabla_{y}^{-} v_{i}^{h} \vee 0\right|\right\} h^{2}
\end{aligned}
$$

The middle sum is $J_{U}\left(v^{h}\right)$, so we need to prove that the last sum equals $\left|v^{h}\right|_{W^{1,1}\left(\Omega^{h}\right)}$. Note that

$$
\left|-\nabla_{x}^{+} v_{i}^{h} \vee 0\right|+\left|\nabla_{x}^{-} v_{i+(1,0)}^{h} \vee 0\right|=\left|-\nabla_{x}^{+} v_{i}^{h} \vee 0\right|+\left|\nabla_{x}^{+} v_{i}^{h} \vee 0\right|=\left|\nabla_{x}^{+} v_{i}^{h}\right|,
$$

so the absolute value of each horizontal and vertical difference in $v^{h}$ is included precisely once in the last sum, so it equals

$$
\sum_{i}\left\{\left|\nabla_{x}^{+} v_{i}^{h}\right|+\left|\nabla_{y}^{+} v_{i}^{h}\right|\right\} h^{2}=\left|v^{h}\right|_{W^{1,1}\left(\Omega^{h}\right)} .
$$

The following lemma corresponds to Lemma 1.4.

LEMMA 5.3.

$$
\left|J_{U}\left(v^{h}\right)-J_{\oplus \ominus}\left(v^{h}\right)\right| \leq h\left|\operatorname{Ext}_{h} v^{h}\right|_{W_{h}^{2,1}\left(2 \Omega^{h}\right)}
$$

where $J_{\oplus \ominus}$ is any discrete variation defined in (1.8).

Proof. We only prove the case for $J_{\oplus \ominus}=J_{++}$. The other cases are the same. 
Note that

$$
\left|\nabla_{x}^{+} v_{i}^{h}\right|^{2}=\left|\nabla_{x}^{+} v_{i}^{h} \vee 0\right|^{2}+\left|\left(-\nabla_{x}^{+}\right) v_{i}^{h} \vee 0\right|^{2},
$$

so we can write $J_{++}\left(v^{h}\right)$ in a similar way to $J_{U}\left(v^{h}\right)$ as

$$
J_{++}\left(v^{h}\right)=\sum_{i \in \Omega^{h}}\left|\left(\begin{array}{r}
-\nabla_{x}^{+} v_{i}^{h} \vee 0 \\
\nabla_{x}^{+} v_{i}^{h} \vee 0 \\
-\nabla_{y}^{+} v_{i}^{h} \vee 0 \\
\nabla_{y}^{+} v_{i}^{h} \vee 0
\end{array}\right)\right| h^{2},
$$

Thus,

$$
\begin{aligned}
\left|J_{U}\left(v^{h}\right)-J_{++}\left(v^{h}\right)\right| & =\left|\sum_{i \in \Omega^{h}}\left\{\left|\left(\begin{array}{r}
-\nabla_{x}^{+} v_{i}^{h} \vee 0 \\
\nabla_{x}^{-} v_{i}^{h} \vee 0 \\
-\nabla_{y}^{+} v_{i}^{h} \vee 0 \\
\nabla_{y}^{-} v_{i}^{h} \vee 0
\end{array}\right)\right|-\left|\left(\begin{array}{r}
-\nabla_{x}^{+} v_{i}^{h} \vee 0 \\
\nabla_{x}^{+} v_{i}^{h} \vee 0 \\
-\nabla_{y}^{+} v_{i}^{h} \vee 0 \\
\nabla_{y}^{+} v_{i}^{h} \vee 0
\end{array}\right)\right|\right\} h^{2}\right| \\
0 & \leq \sum_{i \in \Omega^{h}}\left|\left(\begin{array}{c}
\nabla_{x}^{-} v_{i}^{h} \vee 0-\nabla_{x}^{+} v_{i}^{h} \vee 0 \\
0 \\
\nabla_{y}^{-} v_{i}^{h} \vee 0-\nabla_{y}^{+} v_{i}^{h} \vee 0
\end{array}\right)\right| \\
& \leq \sum_{i \in \Omega^{h}}\left(\left|\nabla_{x}^{-} v_{i}^{h} \vee 0-\nabla_{x}^{+} v_{i}^{h} \vee 0\right|+\left|\nabla_{y}^{-} v^{h} \vee 0-\nabla_{y}^{+} v^{h} \vee 0\right|\right) h^{2} .
\end{aligned}
$$

Because $|a \vee 0-b \vee 0| \leq|a-b|$, we have

$$
\begin{aligned}
\left|J_{U}\left(v^{h}\right)-J_{++}\left(v^{h}\right)\right| & \leq \sum_{i \in \Omega^{h}}\left(\left|\nabla_{x}^{-} v_{i}^{h}-\nabla_{x}^{+} v_{i}^{h}\right|+\left|\nabla_{y}^{-} v_{i}^{h}-\nabla_{y}^{+} v_{i}^{h}\right|\right) h^{2} \\
& \leq h\left|\operatorname{Ext}_{h} v^{h}\right|_{W_{h}^{2,1}\left(2 \Omega^{h}\right)} .
\end{aligned}
$$

We use Lemma 5.2 and Lemma 5.3 to prove the following lemma that corresponds to Lemma 2.1.

Lemma 5.4. There exists a $C>0$ such for any $v \in L^{1}(\Omega)$

$$
J_{U}\left(P_{h} v\right) \leq|v|_{\mathrm{BV}(\Omega)}+C h|\operatorname{Ext} v|_{W^{2,1}(2 \Omega)}
$$

and for any $v^{h}$ defined on $\Omega^{h}$

$$
\left|\operatorname{Int} v^{h}\right|_{\mathrm{BV}(\Omega)} \leq J_{U}\left(v^{h}\right)+C h\left|\operatorname{Ext}_{h} v^{h}\right|_{W_{h}^{2,1}\left(2 \Omega^{h}\right)} .
$$

Proof. The second inequality can be proved by simply combining (2.2) and (5.7).

To prove the first inequality, again we assume that Ext $v \in W^{2,1}(2 \Omega)$, otherwise it is trivial. We apply Lemma 5.3 with $v^{h}=P_{h} v$, then

$$
J_{U}\left(P_{h} v\right) \leq J_{\oplus \ominus}\left(P_{h} v\right)+C h\left|\operatorname{Ext}_{h} P_{h} v\right|_{W_{h}^{2,1}\left(2 \Omega^{h}\right)} .
$$

Then by (2.1) in Lemma 2.1

$$
\begin{aligned}
J_{U}\left(P_{h} v\right) & \leq|v|_{\mathrm{BV}(\Omega)}+C h|\operatorname{Ext} v|_{W^{2,1}(2 \Omega)}+C h\left|\operatorname{Ext}_{h} P_{h} v\right|_{W_{h}^{2,1}\left(2 \Omega^{h}\right)} \\
& =|v|_{\mathrm{BV}(\Omega)}+C h|\operatorname{Ext} v|_{W^{2,1}(2 \Omega)}+C h\left|P_{h} \operatorname{Ext} v\right|_{W_{h}^{2,1}\left(2 \Omega^{h}\right)} \\
& \leq|v|_{\mathrm{BV}(\Omega)}+C h|\operatorname{Ext} v|_{W^{2,1}(2 \Omega)} .
\end{aligned}
$$


The last line follows from (1.25).

Lemma 5.5 is the counterpart of the first inequality (1.32) in Lemma 1.7.

LEMMA 5.5.

$$
J_{U}\left(S_{L} v^{h}\right) \leq J_{U}\left(v^{h}\right)
$$

Proof. The result comes from the symmetry and convexity of $J_{U}$. The proof is exactly the same as the proof for $J_{*}$ in Lemma 1.7.

We note that the proofs of Lemmas 3.1 and 4.1 carry over directly to $J_{U}$, and we obtain the following theorem for the "upwind" discrete variation.

TheOREm 5.1 (Error bounds for upwind scheme). Assume $u$ is the minimizer of the functional

$$
E(v)=\frac{1}{2 \lambda}\|v-g\|_{L^{2}(\Omega)}^{2}+|v|_{\mathrm{BV}(\Omega)}
$$

for $g \in \operatorname{Lip}\left(\alpha, L^{2}(\Omega)\right)$ and $u^{h}$ is the minimizer of the discrete functional

$$
E_{h}\left(v^{h}\right)=\frac{1}{2 \lambda}\left\|v^{h}-P_{h} g\right\|_{L^{2}\left(\Omega^{h}\right)}^{2}+J_{U}\left(v^{h}\right) .
$$

Then

$$
\left\|I_{h} u^{h}-u\right\|_{L^{2}(\Omega)}^{2} \leq C\|g\|_{\operatorname{Lip}\left(\alpha, L^{2}(\Omega)\right)}^{2} h^{\alpha /(\alpha+1)} .
$$

The proof is the same as the proof for the symmetric discrete variation $J_{*}$.

6. Conclusions and extensions. We have proved error bounds for discrete minimizers of two symmetric approximations to the ROF image smoothing model. We remark that our bounds are not "optimal" in an approximation-theory sense, as one can approximate $\operatorname{Lip}\left(\alpha, L^{2}(\Omega)\right)$ functions in $L^{2}(\Omega)$ to order $h^{\alpha}$ and we achieve an error bound of $h^{\alpha /(2 \alpha+2)}$. This bound, coincidentally, coincides with the rate of noise removal using linear methods applied to wavelets, see, e.g., [4]. (The resuls stated there are for functions in $W^{\alpha, 2}(\Omega)$, but they use only inequalities that are equally true for $\operatorname{Lip}\left(\alpha, L^{2}(\Omega)\right)$.)

Somewhat weaker results were proved by the first author in [14] for the functional

$$
J_{h}\left(v^{h}\right)=\frac{1}{2}\left(J_{++}\left(v^{h}\right)+J_{--}\left(v^{h}\right)\right) .
$$

The arguments there exploit the fact that for this particular $J_{h}$

$$
\left|\operatorname{Int} v^{h}\right|_{\mathrm{BV}(\Omega)}=J_{h}\left(v^{h}\right) ;
$$

they also require that $g \in \operatorname{Lip}\left(\beta, L^{1}(\Omega)\right) \cap L^{\infty}(\Omega)$, which implies that $g \in \operatorname{Lip}\left(\alpha, L^{2}(\Omega)\right)$ for $\alpha=\beta / 2$, and they achieve the same convergence rate of $h^{\alpha /(2 \alpha+2)}$.

Finally, similar techniques have been applied to analyze a central difference approximation to $|v|_{\mathrm{BV}(\Omega)}$ in [11]; there the same convergence rate of approximation $O\left(h^{1 / 4}\right)(\alpha=1)$ was achieved, but for quite smooth functions: $g$ is required to be in the Sobolev space $W^{1,2}(\Omega)$, a space that does not contain "images with edges". 


\section{REFERENCES}

[1] C. Bouman and K. Sauer, Bayesian estimation of transmission tomograms using segmentation based optimization, IEEE Trans. Nuclear Science, 39 (1992), pp. 1144-1152.

[2] J. L. Carter, Dual Methods for Total Variation-Based Image Restoration, Ph.D. thesis, 2001, U.C.L.A.

[3] A. Chambolle, An algorithm for total variation minimization and applications, J. Math. Imaging Vision, 20 (2004), pp. 89-97.

[4] A. Chambolle, R. A. DeVore, N.-Y. Lee, and B. J. Lucier, Nonlinear wavelet image processing: Variational problems, compression, and noise removal through wavelet shrinkage, IEEE Trans. Image Processing, 7 (1998), pp. 319-335.

[5] A. Chambolle, S. E. Levine, And B. J. Lucier, Upwind and multiscale methods for ROF image smoothing, preprint, 2009.

[6] T. F. Chan, G. H. Golub, And P. Mulet, A nonlinear primal-dual method for total variation-based image restoration, SIAM J. Sci. Comput., 20 (1999), pp. 1964-1977.

[7] R. A. DeVore, B. Jawerth, And B. J. Lucier, Image Compression through wavelet transform coding, IEEE Trans. Information Theory, 38 (1992), pp. 719-746. Special issue on Wavelet Transforms and Multiresolution Analysis.

[8] R. A. DeVore and G. G. Lorentz, Constructive Approximation, Springer-Verlag, Berlin Heidelberg, 1993.

[9] L. C. Evans And R. F. GaRiepy, Measure theory and fine properties of functions, CRC Press, Boca Raton, FL, 1992.

[10] X. Feng, M. von Oehsen, And A. Prohl, Rate of convergence of regularization procedures and finite element approximations for the total variation flow, Numer. Math., 100 (2005), pp. $441-456$

[11] M.-J. LAI, B. LuCIER, AND J. WANG, The convergence of a central-difference discretization of Rudin-Osher-Fatemi model for image denoising, in Scale Space and Variational Methods in Computer Vision, X.-C.-Tai et al., eds., Lecture Notes in Computer Science, Vol. 5567, Springer Berlin, Heidelberg, 2009, pp. 514-526.

[12] S. Osher And J. A. Sethinan, Fronts propagating with curvature-dependent speed: Algorithms based on Hamilton-Jacobi formulations, J. Comput. Physics, 79 (1988), pp. 12-49

[13] L. I. Rudin, S. Osher, And E. Fatemi, Nonlinear total variation based noise removal algorithms, Physica D., 60 (1992), pp. 259-268.

[14] J. WANG, Error bounds for numerical methods for the ROF image smoothing model, Ph.D. thesis, 2008, Purdue University. 